\title{
An integrated optimisation approach to airport ground operations to foster sustainability in the aviation sector
}

\author{
Michal Weiszer ${ }^{\mathrm{a}, *}$, Jun Chen $^{\mathrm{a}}$, Giorgio Locatelli ${ }^{\mathrm{a}}$ \\ ${ }^{a}$ School of Engineering, University of Lincoln, Brayford Pool, Lincoln, United Kingdom
}

\begin{abstract}
With increasing air traffic, rising fuel costs and tighter environmental targets, efficient airport ground operations are one of the key aspects towards sustainable air transportation. This complex system includes elements such as ground movement, runway scheduling and ground services. Previously, these problems were treated in isolation since information, such as landing time, pushback time and aircraft ground position, are held by different stakeholders with sometimes conflicting interests and, normally, are not shared. However, as these problems are interconnected, solutions as a result of isolated optimisation may achieve the objective of one problem but fail in the objective of the other one, missing the global optimum eventually. Potentially more energy and economic costs are thus required. In order to apply a more systematic and holistic view, this paper introduces a multi-objective integrated optimisation problem incorporating the newly proposed Active Routing concept. Built with systematic perspectives, this new model combines several elements: scheduling and routing of aircraft, 4-Dimensional Trajectory (4DT) optimisation, runway scheduling and airport bus scheduling. A holistic economic optimisation framework is also included to support the decision maker to select the economically optimal solution from a Pareto front of technically optimal solution. To solve this problem, a multi-objective genetic algorithm is adopted and tested on real data from an international hub airport. Preliminary results show that the proposed approach is able to provide a systematic framework so that airport efficiency, environmental assessment and economic analysis could all be explicitly optimised.
\end{abstract}

Keywords: Airport operations, Environmental impact, Ground movement, multi-objective optimisation

\footnotetext{
* Corresponding author

Email addresses: mweiszer@lincoln.ac.uk (Michal Weiszer), juchen@lincoln.ac.uk (Jun Chen), glocatelli@lincoln.ac.uk (Giorgio Locatelli)
} 


\section{Introduction}

Global air traffic is continuing to grow steadily and the 3.1 billion airline passengers carried in 2013 are forecasted to double to about six billion by 2030 [1]. By that time, many airports will reach their maximum capacity resulting in a great pressure to fully utilise the available resources and the need for efficient ground operations. Furthermore, the global effort to meet ambitious environmental targets such as reaching an emission-free airport ground movement in Europe by 2050 [2], together with rising fuel costs, push the airlines to cut fuel consumption as much as possible. Advances in research in the last decades have seen improvement in the fuel efficiency and mitigation of environmental impact for new aircraft due to innovative design [3] or the application of alternative fuels [4, 5, 6]. However, in addition to technological developments in maximising energy utilisation, there is a considerable potential to achieve the same objective by optimising operational procedures at airports, which is still untapped fully.

Previously, different information (e.g. landing time, pushback time or aircraft ground position) were possessed by different stakeholders with limited sharing. However, with the abovementioned challenges imposed on airports, this approach cannot be sustained in the future. This was recognized by Eurocontrol with the introduction of the Airport Collaborative Decision Making (A-CDM) concept [7]. The core idea of A-CDM is the cooperation and real-time data sharing between airport operators, aircraft operators, ground handlers and air traffic control in order to reduce delays, improve the predictability of events and optimise the utilisation of resources. In line with A-CDM concept, optimisation of different airport ground problems such as ground movement, runway scheduling, gate assignment and scheduling of ground services need to be treated in a more integrated and coordinated manner instead of current isolated practices, to fully appreciate the same positive effects given by A-CDM.

Previous research on airport ground operations mostly focused on individual sub problems. A number of papers have been published on runway scheduling problem. The objective is often expressed as a minimisation of delay, the number of changes compared to First-come-first-served (FCFS) sequence, makespan or their combination. A wide range of exact and heuristic methods employed to solve this problem include dynamic programming [8], hybrid tabu search [9, 10], genetic algorithm [11] and heuristics [12]. A detailed review of recent research on runway scheduling problem can be found in [13].

Previous papers on ground movement problem mostly focused on minimisation of the total taxi time or other time related objectives [14]. Minimisation of the total taxi time is the main goal of the genetic algorithm proposed by Pesic et al. [15], mixed integer linear programming formulation used in [16, 17] or a graph-based approach utilised in [18] or [19]. Deviations from the scheduled time of departure or arrival are penalized in [20,21]. A combination of time related objectives is minimised in [22].

Recently, a few researchers started to consider also fuel consumption as a objective for the ground movement problem. Papers focused on the stand holding problem [23, 24, 25] take the fuel consumption into account indirectly, maximising the time an aircraft spends at the stand, with their engines off, rather than taxiing. Multi-objective optimisation has been employed by Ravizza et al. [26] to simultaneously minimise taxi time as well as fuel consumption. Their approach combines a routing and scheduling algorithm [18] with the Population Adaptive based Immune Algorithm (PAIA) [27] in search of the trade-off between the total taxi time and fuel consumption expressed as a fuel consumption index. The following work [28] introduced a fast heuristic procedure for speed profile optimisation to speed up the search. Results in [26, 27, 28] indicated that the fastest schedule normally leads to higher fuel burn due to heavy acceleration required to achieve short taxi time.

Only a few papers considered ground movement and runway scheduling as an integrated and interconnected problem. Deau et al. [29, 30] proposed a two-stage approach in which a branch and bound algorithm was used, in the first stage, to find the best runway sequence regarding the deviation from assigned slots and then, in the second stage, a genetic algorithm was applied to find a solution for the ground movement problem minimising the difference from the target times resulting from the runway sequence found in the first stage. A mixed integer linear programming formulation by Clare and Richards [31] minimises a weighted sum of taxi time and distance related objectives with respect to runway scheduling constraints. Frankovich and Bertsimas [32] introduced an integer programming formulation for selecting a runway configuration, assigning flights to runways and determining their sequence, and after solving these and fixing them, deter- 
mining the gate-holding duration of departures and routing of flights on the airport surface with the aim of minimising delays.

Research on the optimisation of ground services includes scheduling of airport buses [33], optimisation of luggage handling process [34], or scheduling of other services [35] such as fuelling [36], catering [37], cleaning, water and sanitation processes. As pointed out in [38, 33, 35], optimisation of ground services shares similar characteristics. As a result, and due to the fact that the particular airport under investigation in this study does not have gates, only stands, in this paper we focus only on the scheduling of airport buses which is an example of ground service optimisation problems. Although gate assignment has a direct impact on ground movement through the location of gates/stands assigned to flights [39], its planning is normally carried out at a tactical level usually for the whole day. Since ground movement, runway scheduling and scheduling of ground services requires operational planning, gate assignment is not main focus of this work. It is worth pointing out that these optimisation problems are closely interrelated with each other, for example, a runway sequence determines times at which aircraft have to start/finish their taxi and subsequently the schedule of ground services needed at the gates.

Recently, this kind of optimisation problems has been introduced in [40] as multi-component optimisation problems, which are common in transportation research [41, 42, 9]. As shown in [40, 43], optimisation of multi-component problems in an isolated manner may not find globally optimal solutions, since solution for one problem can fail in the objective of the other one and thus miss the global optimum. Furthermore, these problems are not only difficult to solve in their own right, but even more so when combined, due to the interdependence among them. The proposed approach in this paper follows the same line of research. Legitimately, this type of problems with different stakeholders and objective functions can be tackled more easily with a multi-objective optimisation approach [43], in which each objective can be addressed appropriately. Furthermore, as the result of multi-objective optimisation is a set of solutions, under unprecedented events, the decision maker will have more readily available alternatives as backup plans without sacrificing too much cost or other resources. Finally, improving predictability of events by implementing A-CDM concept both in operation and optimisation means that previously conservative planning can now be reviewed in order to further improve the airport capacity, decrease excessive waiting time, avoid fuel-intensive speed profiles or requirement of extra resources.

In the light of the above discussions, in this paper, we propose to use a multi-objective genetic algorithm framework, namely the Non-dominated Sorting Genetic Algorithm-II (NSGA-II) [44], which considers several elements: ground movement problem, runway scheduling and scheduling of airport buses in a more holistic manner. This integrated multi-objective approach incorporating the optimal 4-Dimensional Trajectory (4DT) [27] enables the investigation of the trade-off between different objectives and, assuming the A-CDM system is in place, facilitates more precise control of the taxiing aircraft in order to take full advantage of optimised scheduling. Furthermore, a holistic economic optimisation framework is introduced in this paper to support the decision maker in selecting the most cost-effective solution from a Pareto front of optimal solutions. The main contributions of this paper can be summarized as follows:

- The proposed integrated multi-objective approach optimises ground movement problem, runway scheduling and scheduling of airport buses simultaneously with respect to different objectives, in particular fuel consumption, which was not considered in previous studies. It is worth mentioning, that runway scheduling and ground movement problem are in itself multi-objective problems.

- By using the proposed integrated multi-objective approach, a comprehensive comparative study is conducted by choosing the representative different solution approaches found in the literature with respect to different objectives.

- The introduced economic optimisation framework represents a general framework in selection of a sustainable solution to the airport problems in view of an economic perspective.

The rest of the paper is organised as follows. Section 2 provides details about individual components of the integrated model, including runway scheduling, ground movement problem and scheduling of airport buses. The integrated solution method and the proposed evolutionary algorithm framework is described in 
We denote the waiting time $w_{i}$ of the departing aircraft $i \in D$ : section. Finally, conclusions are drawn in Section 5.

\section{Problem description}

\subsection{Runway scheduling} aircraft still on the ground rather than airborne arriving aircraft. departing on the same SID. until it is safe to take-off:

Section 3. A set of computational experiments are carried out using data instances from Doha International Airport in Section 4; results of the isolated approach and the proposed method are also compared in this

In this paper, the integrated optimisation problem of airport ground operations consists of three subproblems: 1) runway scheduling, 2) ground movement problem, 3) bus scheduling problem. The individual sub-problems are described in the next sections using nomenclature given in Table 1.

The runway scheduling problem generally consists of the Aircraft Landing Problem (ALP) and the Aircraft Take-off Problem (ATP) with the aim to find the optimal sequence and scheduled landing time or take-off time of aircraft at the given runways with respect to the given objective functions and constraints. In this paper, we consider only ATP, as from the practical point of view, it is easier to control taking-off

The minimum time interval between aircraft landing or taking-off constrains the throughput of the runway. The enforced separations between aircraft are due to wake vortices and in-flight separation constraints. Landing or taking-off aircraft create wake vortices which have to dissipate before another aircraft can safely use the runway. The strength of wake vortices and thus separation depends on the type and weight of aircraft. A larger separation is required whenever a light aircraft follows a larger and heavier aircraft, as it creates stronger wake vortices. Furthermore, if aircraft use standard instrument departure routes (SIDs), additional separation is needed to ensure correct in-flight separation [13]. The use of SIDs is not considered in this paper as the Doha international airport under consideration does not have SIDs established. However, the SIDs related separation can be easily taken into account as described later in this section.

The formal definitions for runway scheduling presented in this paper are as follows. Let $M=(A \cup D)$ be the set of total $|M|=m$ arriving aircraft $A$ and departing aircraft $D$. The wake vortex separations are estimated using minimum separation distance, runway occupancy time and average velocities for approach/climb as described in [45] and are given in Table 2. Then, we define $V\left(v_{i}, v_{j}\right)$ to be the function to return the wake vortex separations from Table 2 for weight categories $v_{i}$ and $v_{j}$ of leading aircraft $i$ and trailing aircraft $j$. The wake vortex separations used in this paper satisfy the triangle inequality $V\left(v_{i}, v_{j}\right)+V\left(v_{j}, v_{e}\right) \geq V\left(v_{i}, v_{e}\right)$ for aircraft taking off in the order of $i, j, e$. In case of established SIDs, the related separations can be considered by a similar function and a table as $V\left(v_{i}, v_{j}\right)$ and Table 2 for aircraft

Let $r_{i}$ be the actual landing time for aircraft $i \in A$ and take-off time for aircraft $i \in D$. For arriving aircraft, $r_{i}$ is given, while for departing aircraft it can be calculated as follows. Let $d_{i}$ denote the time the departing aircraft $i \in D$ arrived at the runway holding point, then it can take-off immediately, i.e. $d_{i}=r_{i}$ if there is enough time elapsed from landing/take-off time $r_{i-1}$ of the previous aircraft $i-1$ to comply with separation given by $V\left(v_{i}, v_{i-1}\right)$, otherwise, the departing aircraft $i$ has to wait at the runway holding point

$$
r_{i}= \begin{cases}d_{i} & \text { if } d_{i}-r_{i-1} \geq V\left(v_{i}, v_{i-1}\right), \\ d_{i}+w_{i} & \text { otherwise. }\end{cases}
$$

$$
w_{i}= \begin{cases}0 & \text { if } d_{i}-r_{i-1} \geq V\left(v_{i}, v_{i-1}\right), \\ V\left(v_{i}, v_{i-1}\right)-\left(d_{i}-r_{i-1}\right) & \text { otherwise }\end{cases}
$$


Table 1: Nomenclature.

Description

\begin{tabular}{|c|c|c|c|}
\hline$g_{1}$ & The total time objective & $\tau($ origin, destination $)$ & $\begin{array}{l}\text { Travelling time of the bus be- } \\
\text { tween origin and destination }\end{array}$ \\
\hline$g_{2}$ & The fuel consumption objective & $N$ & Set of active bus trips \\
\hline$g_{3}$ & $\begin{array}{l}\text { The bus scheduling cost objec- } \\
\text { tive }\end{array}$ & $n$ & The number of bus trips \\
\hline$M$ & Set of all aircraft & $G=(O, P)$ & Vehicle scheduling network \\
\hline$m$ & The number of all aircraft & $O$ & $\begin{array}{l}\text { Set of nodes on the vehicle } \\
\text { scheduling network }\end{array}$ \\
\hline$A$ & Set of arriving aircraft & $P$ & $\begin{array}{l}\text { Set of arcs on the vehicle schedul- } \\
\text { ing network }\end{array}$ \\
\hline$D$ & Set of departing aircraft & $c$ & Cost of arc \\
\hline$V\left(v_{i}, v_{j}\right)$ & $\begin{array}{l}\text { Function to calculate the wake } \\
\text { vortex separations for weight } \\
\text { categories } v_{i} \text { and } v_{j} \text { of leading } \\
\text { aircraft } i \text { and following aircraft } j\end{array}$ & $b$ & $\begin{array}{l}\text { Variable determining if arc is } \\
\text { covered by a bus }\end{array}$ \\
\hline$v$ & Weight category & $k, l$ & Bus trip index \\
\hline$i, j, e$ & Aircraft index & $p o p_{\max }$ & Maximum number of generations \\
\hline$r$ & $\begin{array}{l}\text { Actual landing time for arriving } \\
\text { aircraft/take-off time for depart- } \\
\text { ing aircraft }\end{array}$ & pop number & The current generation index \\
\hline$d$ & $\begin{array}{l}\text { Arrival time of the departing air- } \\
\text { craft at the runway holding point }\end{array}$ & $c^{a}$ & The total strategic cost \\
\hline$w$ & Waiting time at the runway & $c^{\text {fuel }}$ & Fuel cost \\
\hline$t^{r w y}$ & The total runway delay & $C^{\text {total }}$ & The total cost \\
\hline$f^{r w y}$ & The total runway fuel & $R$ & Rated output of aircraft \\
\hline$\phi_{v_{i}}, \phi_{v_{i}}^{i d l e}$ & $\begin{array}{l}\text { Calculated and idle fuel flow for } \\
\text { weight category } v_{i} \text {, respectively }\end{array}$ & $h$ & Weight \\
\hline$\delta$ & $\begin{array}{l}\text { Safety time distance between } \\
\text { taxiing aircraft }\end{array}$ & $a, a_{\max }$ & $\begin{array}{l}\text { acceleration and maximum accel- } \\
\text { eration of aircraft respectively }\end{array}$ \\
\hline$y$ & $\begin{array}{l}\text { Integer representing the speed } \\
\text { profile }\end{array}$ & $\eta$ & Thrust level \\
\hline$q$ & The shortest taxi route & $T h r$ & Thrust \\
\hline$s$ & Stand & weight & Weight of aircraft \\
\hline$T\left(q_{i}, y_{i}\right)$ & $\begin{array}{l}\text { Travel time of aircraft } i \text { taxiing } \\
\text { on route } q_{i} \text { for given speed profile } \\
y_{i}\end{array}$ & $F R$ & Rolling resistance \\
\hline$x$ & The pushback time & $p_{a r r}$ & $\begin{array}{l}\text { Arrival time of buses after arriv- } \\
\text { ing aircraft came to a stand }\end{array}$ \\
\hline$z$ & The arrival time to the stand & $p_{e m b}$ & $\begin{array}{l}\text { Embarking/disembarking time } \\
\text { of passengers }\end{array}$ \\
\hline$F\left(q_{i}, y_{i}, v_{i}\right)$ & $\begin{array}{l}\text { The amount of fuel burned for } \\
\text { aircraft } i \text { of weight category } v_{i} \\
\text { during taxiing on the route } q_{i} \text { fol- } \\
\text { lowing the speed profile } y_{i}\end{array}$ & $p_{\text {dep }}$ & $\begin{array}{l}\text { Departure time of buses before } \\
\text { the pushback time of departing } \\
\text { aircraft }\end{array}$ \\
\hline$t^{t a x i}$ & The total taxi time & $p_{\text {headway }}$ & Headway between buses \\
\hline$f^{t a x i}$ & $\begin{array}{l}\text { The total fuel burned during } \\
\text { ground movement }\end{array}$ & $s p$ & Average speed of buses \\
\hline
\end{tabular}


Table 2: Separations in seconds between departing (D) and arriving (A) flights for weight classes: Heavy (H), Large (L), Small (S) [45].

\begin{tabular}{cccccccc}
\hline & \multicolumn{7}{c}{ Trailing $\left(v_{j}\right)$} \\
\cline { 3 - 8 } & & A-H & A-L & A-S & D-H & D-L & D-S \\
A-H & 96 & 157 & 207 & 60 & 60 & 60 \\
& A-L & 60 & 69 & 123 & 60 & 60 & 60 \\
A-S & 60 & 69 & 82 & 60 & 60 & 60 \\
& D-H & 60 & 60 & 60 & 96 & 120 & 120 \\
D-L & 60 & 60 & 60 & 60 & 60 & 60 \\
D-S & 60 & 60 & 60 & 60 & 60 & 60 \\
\hline
\end{tabular}

The objective of the runway scheduling is to minimise the total runway delay $t^{r w y}$ and the total runway fuel $f^{r w y}$ burned by aircraft while waiting to take-off which depends on the delay $w_{i}$ and idle fuel flow $\phi_{v_{i}}^{\text {idle }}$ specified for the weight category $v_{i}$ :

$$
t^{r w y}=\sum_{i=1}^{D} w_{i}
$$

$$
f^{r w y}=\sum_{i=1}^{D} w_{i} \cdot \phi_{v_{i}}^{\text {idle }}
$$

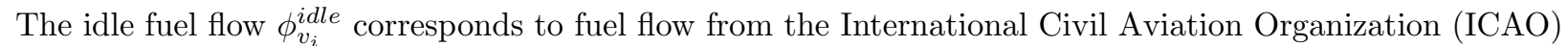
engine database for $5 \%$ of full power thrust of the representative aircraft, as explained in Section 2.2.

\subsection{Ground movement}

The aim of the ground movement problem is to route aircraft from source to destination locations, i.e. from runway to gate/stand and vice versa in a time and fuel efficient manner, respecting routes of other aircraft while preventing conflicts between them.

In this paper, we follow a concept introduced in [26, 27], which in the light of previous research can be called Active Routing (AR) in acknowledge of the fact that the optimised 4DTs for ground movement, consisting of three spatial dimensions and time as the fourth dimension, are seamlessly embedded within the optimisation of routes and schedules. AR consists of two parts: firstly, the routing and scheduling problem aims to find a set of optimal routes and schedules for arriving or departing aircraft, and the 4DT optimisation problem focuses on finding a set of unimpeded multi-objective optimal speed profiles for the routes from the first part. In this paper, we use the shortest path algorithm as a route optimisation method. However, any routing method, such as the $k$-QPPTW [26], could be used instead.

Speed profiles are used in this paper to represent $4 \mathrm{DTs}$, since not all dimensions are required as aircraft's movement are bounded by taxiways. In this case, it is sufficient to completely define their position in time with routes and speed profiles. In order to further reduce the complexity of the speed profile optimisation problem, the route of an aircraft is further divided into larger segments, each containing several edges as shown in Fig. 1. For example, several consecutive straight edges typically form one straight segment. The turning segment consists of consecutive edges between which have an angle of at least 30 degrees. The maximum speed on straight segments is restricted to maximum 30 knots $\left(15.43 \mathrm{~m} \cdot \mathrm{s}^{-1}\right)$ and turning speed is set to a constant speed of 10 knots $\left(5.14 \mathrm{~m} \cdot \mathrm{s}^{-1}\right)$, similarly as in $[27,26,28]$. Subsequently, each straight segment is divided into four parts, corresponding to four different aircraft taxiing phases (acceleration, travelling at constant speed, braking and rapid braking with maximum deceleration) with a typical taxiing behaviour as can be seen in Fig. 2). Furthermore, the maximum acceleration and deceleration rate $a_{\max }$ is set to $0.98 \mathrm{~m} \cdot \mathrm{s}^{-2}$ for passenger comfort [46]. The resulting piece-wise linear speed profile can be described using four free variables (acceleration, length of acceleration phase, length of constant speed phase, length of rapid deceleration phase) which define a unique speed profile over a segment. By searching for values of 


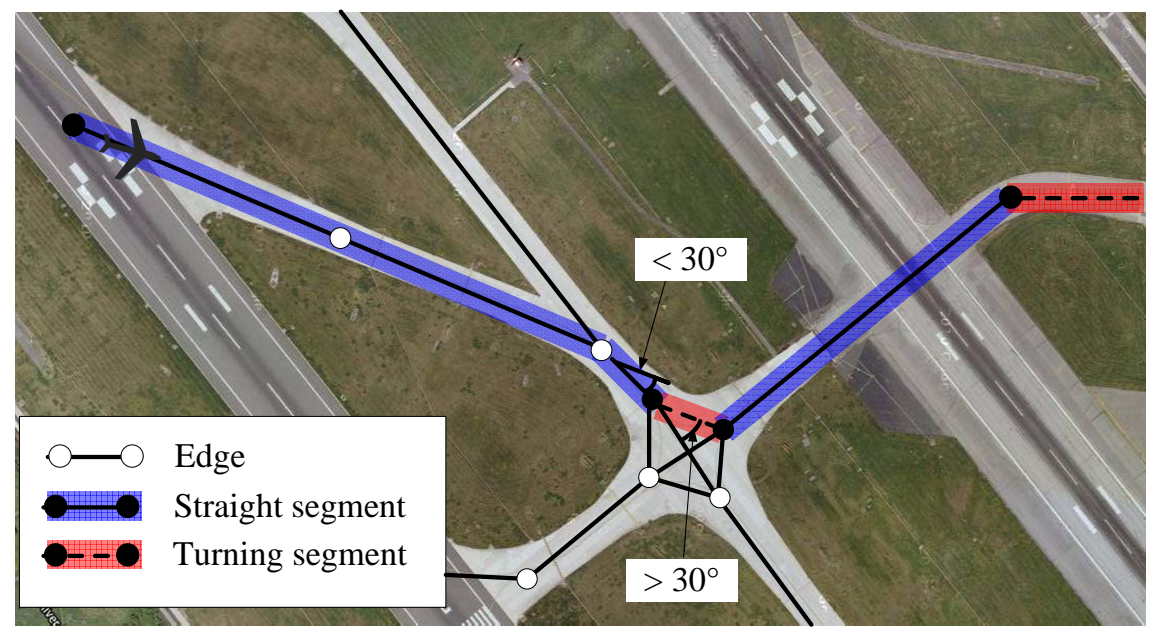

Figure 1: An example of one route of an aircraft on taxiways, represented by segments consisting of edges.

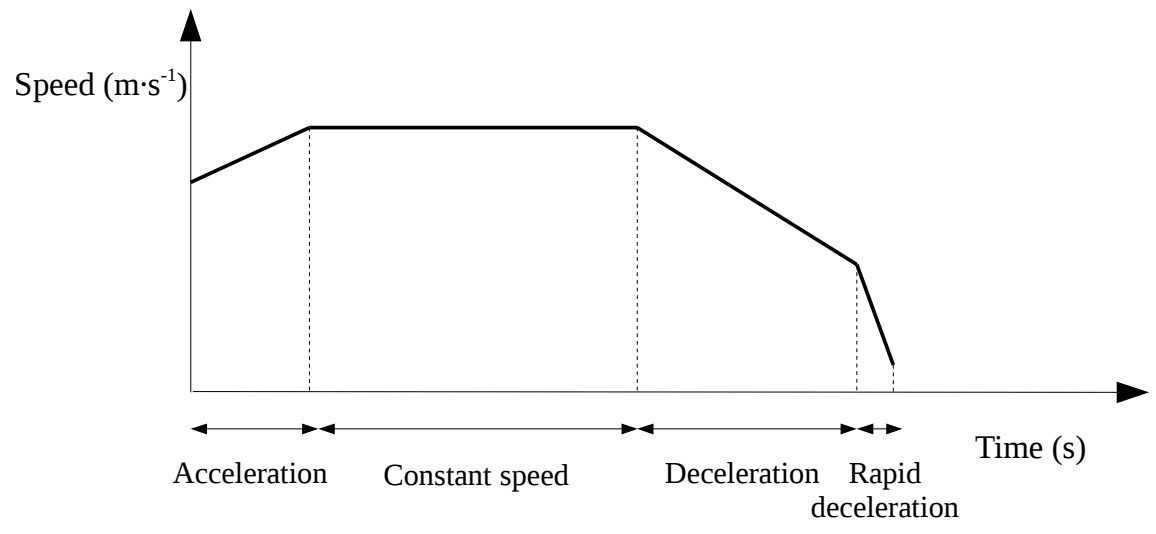

Figure 2: An illustration of a speed profile on a straight segment, divided into four phases.

these four variables, one can explore different speed profiles with different taxi time and fuel consumption. The heuristic described in [28] is employed to find optimised speed profiles.

Fuel consumption corresponding to a speed profile is calculated as follows. As mentioned above, four phases are defined for a straight segment: acceleration, constant speed, braking and rapid braking. Firstly, thrust levels for each phase are established, which for the phase of braking and rapid braking the thrust levels are assumed to be $5 \%$ of full rated power whereas for turning the thrust level is set to 7\% [47]. For other phases, the thrust levels are estimated as a ratio of calculated thrust $T h r$ and maximum power output $R$ of the engine:

$$
\eta=\frac{T h r}{R}
$$

Thrust $T h r$ is a sum of acceleration force, calculated as product of aircraft's weight weight and acceleration $a$, and rolling resistance force $F R$ :

$$
T h r=\text { weight } \cdot a+F R
$$

The fuel flow $\phi_{v_{i}}$ corresponding to the thrust level $\eta$ is obtained by linear interpolation/extrapolation using reported fuel flows from ICAO database at $7 \%$ and $30 \%$ similarly as in [47]. Finally, the fuel consumption for the segment is calculated by multiplication of fuel flow for the specific phase and the time spent in this state. For details of this approach, interested readers are referred to [46]. 


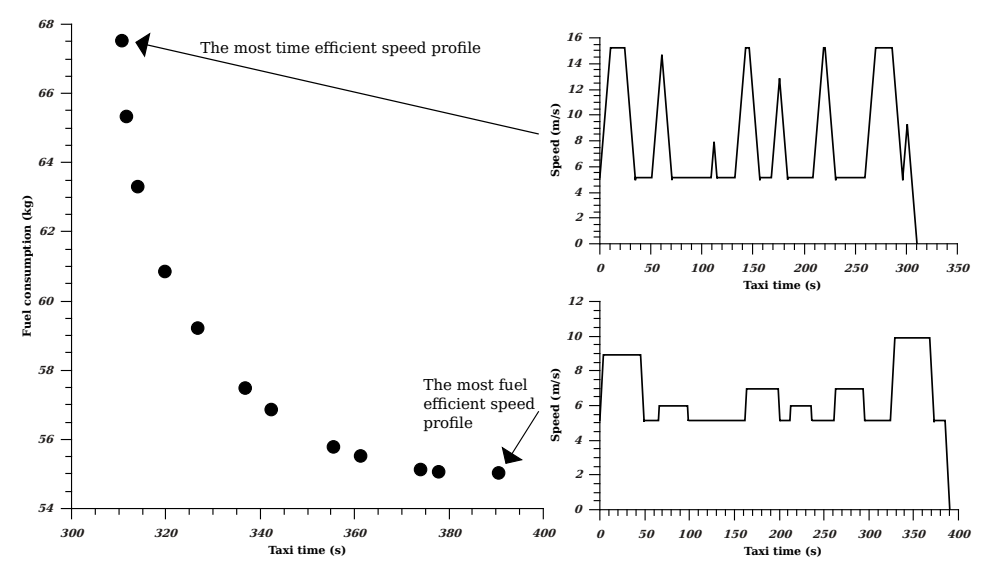

Figure 3: A typical Pareto front of optimal speed profiles for the shortest route between the gate and the runway with speed profiles shown (left), the most time and fuel efficient speed profiles corresponding to solutions indicated by arrows (right).

As the shortest path between gate and runway can be generated before on-line optimisation, the optimised speed profiles for each route $q_{i}$ and weight category $v_{i}$ can be pre-computed as well, stored in a database (lookup table) as a Pareto front and then retrieved during the on-line optimisation in order to save computational time. As can be seen in the example of the Pareto front for the shortest route in Fig. 3, only non-dominated optimised speed profiles are saved in the database.

Given the route of the aircraft and time needed to travel from origin to destination depending on the chosen speed profile, some delay may be added in order to prevent conflicts between taxiing aircraft. A conflict is prevented by maintaining a safe time distance $\delta=12 \mathrm{~s}$ between aircraft (which corresponds to approximately $62 \mathrm{~m}$ at taxiing speed 10 knots, similarly as in [15]).

Let $y_{i}$ be an integer representing the speed profile of aircraft $i$ from the Pareto front of efficient speed profiles retrieved from the database for the shortest route $q_{i}$ from the runway to the stand $s_{i}$ for arriving aircraft $i \in A$, or vice versa for departing aircraft $i \in D$. We define a function $T\left(q_{i}, y_{i}\right)$ which returns travel time of aircraft $i$ taxiing on route $q_{i}$ for given speed profile $y_{i}$, including delay to prevent taxiing conflicts. Function $F\left(q_{i}, y_{i}, v_{i}\right)$ is defined to return the amount of fuel burned for aircraft $i$ of weight category $v_{i}$ during taxiing on the route $q_{i}$ following the speed profile $y_{i}$.

Then, the objective of the ground movement problem is to minimise the total taxi time $t^{\text {taxi }}$ and the total fuel $f^{\text {taxi }}$ burned during ground movement:

$$
t^{t a x i}=\sum_{i=1}^{M} T\left(y_{i}\right)
$$

$$
f^{\text {taxi }}=\sum_{i=1}^{M} F\left(y_{i}\right)
$$

\subsection{Bus scheduling}

The third objective is related to the airport bus scheduling problem. In addition to gates, an airport usually has a number of remote stands which are located on the apron. For each aircraft parked at the stand, one or several buses are needed to transfer passengers from the aircraft to the terminal or vice versa. This creates a scheduling problem which is similar to the vehicle scheduling problem (VSP) encountered in public transportation. The airport bus scheduling problem is relevant to almost all airports with remote stands as passengers usually cannot just walk to/from the terminal. The difficulty in solving the problem grows for busier airports and therefore more relevant to bigger airports. Nevertheless, as mentioned in Section 1, 
scheduling of different ground services bears similarities, and therefore airport bus scheduling problem can be regarded as an example of ground service optimisation problems.

The VSP considered in this paper is formulated as the Single-depot vehicle scheduling problem (SDVSP) which is a special case of VSP with a single depot and single type of vehicles. The goal of the VSP in general is to find a set of feasible vehicle schedules which assign trips to vehicles such that:

- every active trip is covered by exactly one vehicle,

- each vehicle performs a feasible sequence of compatible trips, or none at all, and

- the overall cost of the schedules is minimised.

A bus journey which is used to carry passengers is named active trip, otherwise it is called a deadheading trip. Two trips are said to be compatible if they can be carried out consecutively by the same vehicle. Each aircraft $i$ parked at stand $s_{i}$ gives rise to active trips between the stand $s_{i}$ and the terminal (or in the opposite direction) as follows. Similarly, as for runway scheduling, aircraft are categorized into weight categories according their weight. It is assumed that small category aircraft needs one bus to transfer all its passengers, large category aircraft two and heavy category requires three buses. This assumption is based on the fact, that airports usually use a specialised bus from Cobus which has a capacity of about 100 passengers. The way the trips are generated is in general prescribed by airport management and is different for arriving and departing flights [33]. Usually, if multiple buses are needed to serve an aircraft, trips do not start at the same time, but rather they are shifted, as passengers first embark/disembark the first bus, then the second, etc. Let $x_{i}$ be the pushback time from the stand $s_{i}$ for departing aircraft $i \in D$ and $z_{i}$ the arrival time to the stand $s_{i}$ for arriving aircraft $i \in A$. In this paper, the following time requirements are assumed: for arriving flights $i \in A$, the first and the second bus (if any) are required to be present at the stand $s_{i}$ no later than $p_{a r r}=1$ minute after the arrival time $z_{i}$ at the stand $s_{i}$ of aircraft $i$ due to the fact that usually two stairs are used at the same time to disembark passengers from the aircraft. The third bus has to arrive $p_{\text {arr }}+p_{\text {headway }}=4$ minutes after $z_{i}$, where the headway $p_{\text {headway }}=3$ minutes. For departing flights $i \in D$, the first bus must finish disembarking passengers $p_{\text {dep }}=2$ minutes before the pushback time $x_{i}$, the second bus has to leave $p_{\text {dep }}+p_{\text {headway }}=5$ minutes, and the third bus $p_{\text {arr }}+2 \times p_{\text {headway }}=8$ minutes before $x_{i}$. The time needed for the passengers to embark/disembark the bus $p_{\text {emb }}$ is set to 10 minutes. By adding travelling time of the bus specified by the function $\tau$ (origin, destination) and embarking/disembarking time $p_{\text {emb }}$ to $z_{i}$ for arriving aircraft or $x_{i}$ for departing aircraft, respectively, we can determine the time the bus is at the terminal. The travelling time of the bus $\tau$ (origin, destination) is calculated using the distance and an average speed $s p=50 \mathrm{~km} \cdot \mathrm{h}^{-1}$. This way, a set of active trips $N=\{1,2, \ldots, n\}$ can be defined by considering all flights $i \in M$. In addition to these trips, each bus which is used during the day has to perform a pull-out trip from the depot at the beginning of the day and a pull-in trip at the end of the day.

The SDVSP can be defined using the formulation presented in [48] as follows. Let $G=(O, P)$ be the vehicle scheduling network with set of nodes $O$ representing start and end locations of each trip and set of $\operatorname{arcs} P$ corresponding to trips. Each arc has a cost $c_{k l}$ associated with it which corresponds to time needed for the bus to get from origin to destination. To represent costs for using a vehicle, arcs from or to a depot have a large fixed cost penalty set to 10,000 minutes. We introduce a decision variable $b_{k l}$ which is equal to 1 if a vehicle covers trip $k$ directly after trip $l$, and $b_{k l}=0$ otherwise. The goal of SDVSP is to minimise the objective function:

$$
\min g_{3}=\sum_{(k, l) \in P} c_{k l} b_{k l}
$$

Subject to: 


$$
\begin{array}{lr}
\sum_{l:(k, l) \in P} b_{k l}=1 & \forall k \in N \\
\sum_{k:(k, l) \in P} b_{k l}=1 & \forall l \in N \\
b_{k l} \in\{0,1\} & \forall(k, l) \in P
\end{array}
$$

The constraints 8 and 9 ensure that each trip is assigned to exactly one predecessor and one successor. Finally, the bus scheduling costs are determined by transforming the abovementioned mathematical model to a linear assignment problem and solving it by the algorithm of [49] with the generated trips as an input.

\subsection{Integrated optimisation problem}

The abovementioned optimisation sub-problems are combined into the integrated optimisation problem with the objective functions:

- $g_{1}$ : the total time,

- $g_{2}$ : fuel consumption,

- $g_{3}$ : bus scheduling cost.

As can be seen in Equation 11, $g_{1}$ corresponds to the sum of total taxi time $t^{\text {taxi }}$ and runway delay $t^{r w y}$. The second objective $g_{2}$, as can be seen in Equation 12, relates to the fuel consumption during ground movement $f^{\text {taxi }}$ and waiting for the take-off $f^{r w y}$. The third objective $g_{3}$ is equal to the bus scheduling costs as defined in Equation 7.

$$
\begin{gathered}
g_{1}=t^{t a x i}+t^{r w y} \\
g_{2}=f^{t a x i}+f^{r w y}
\end{gathered}
$$

The three sub-problems are interconnected by decision variables, namely the pushback time for departing aircraft $x_{i}$ and the speed profile $y_{i}$ for all aircraft, from which all other input parameters can be derived as explained above and illustrated in Fig 4 . The decision variable $x_{i}$ is an integer value in the range from -300 to 300, representing seconds before/after the baseline time given as input flight schedule. The range of values for decision variable $y_{i}$ is from 1 to 12 , due to the specifications of the pre-computed database which stores 12 different Pareto optimal speed profiles. The value of 1 represents the most time-efficient (fastest) speed profile whereas 12 corresponds to the most fuel efficient one.

\section{A multi-objective integrated framework incorporating economic optimisation}

\subsection{Integrated solution method}

In order to optimise the objective functions of the integrated optimisation problem stated in Section 2 , a multi-objective evolutionary framework is proposed in this section.

The solution framework for the integrated optimisation problem is based on the implementation of Fast Non-dominated Sorting Genetic Algorithm (NSGA-II) [44] which is a well-known evolutionary algorithm adapted for multi-objective optimization. In evolutionary algorithms, solutions to a problem are represented as individuals in a population. Each individual consists of a set of genes, where each gene corresponds to a decision variable. As stated in Section 2.4, the decision variables are the pushback time $x_{i}$ for departing aircraft $i \in D$ and the speed profile $y_{i}$ for all aircraft $i \in M$.

The basic structure of our algorithm is outlined in Algorithm 1. Firstly, the initial population is generated by creating individuals with random values of the genes. The initial population is evaluated in lines $2-4$. In the next step, individuals with the high fitness values, i.e. with low objective function values, are selected for reproduction. New individuals are created by applying a 2-point crossover to two parent individuals. Next, 


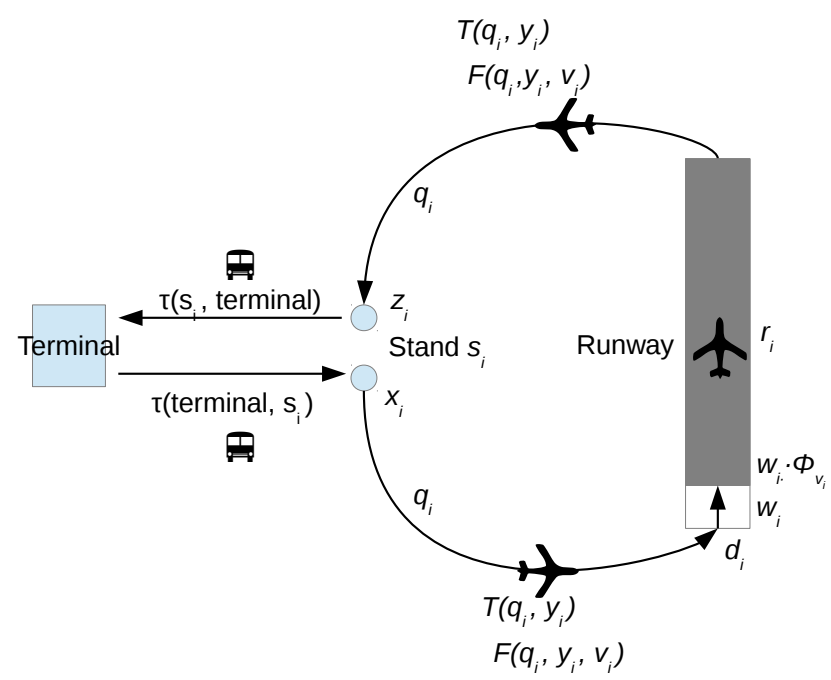

Figure 4: Schematic diagram of airport ground operations showing the relationship between parameters of the optimisation framework.

for each individual a mutation is performed according to the mutation rate. During the mutation, the value of one gene is randomly changed. The individual is then evaluated and objectives $g_{1}, g_{2}, g_{3}$ are calculated. The loop in lines $3-15$ is repeated until the maximum number of generations pop $_{\max }$ is reached. The result of the evolutionary algorithm is then a set of individuals which form the Pareto front of optimal solutions.

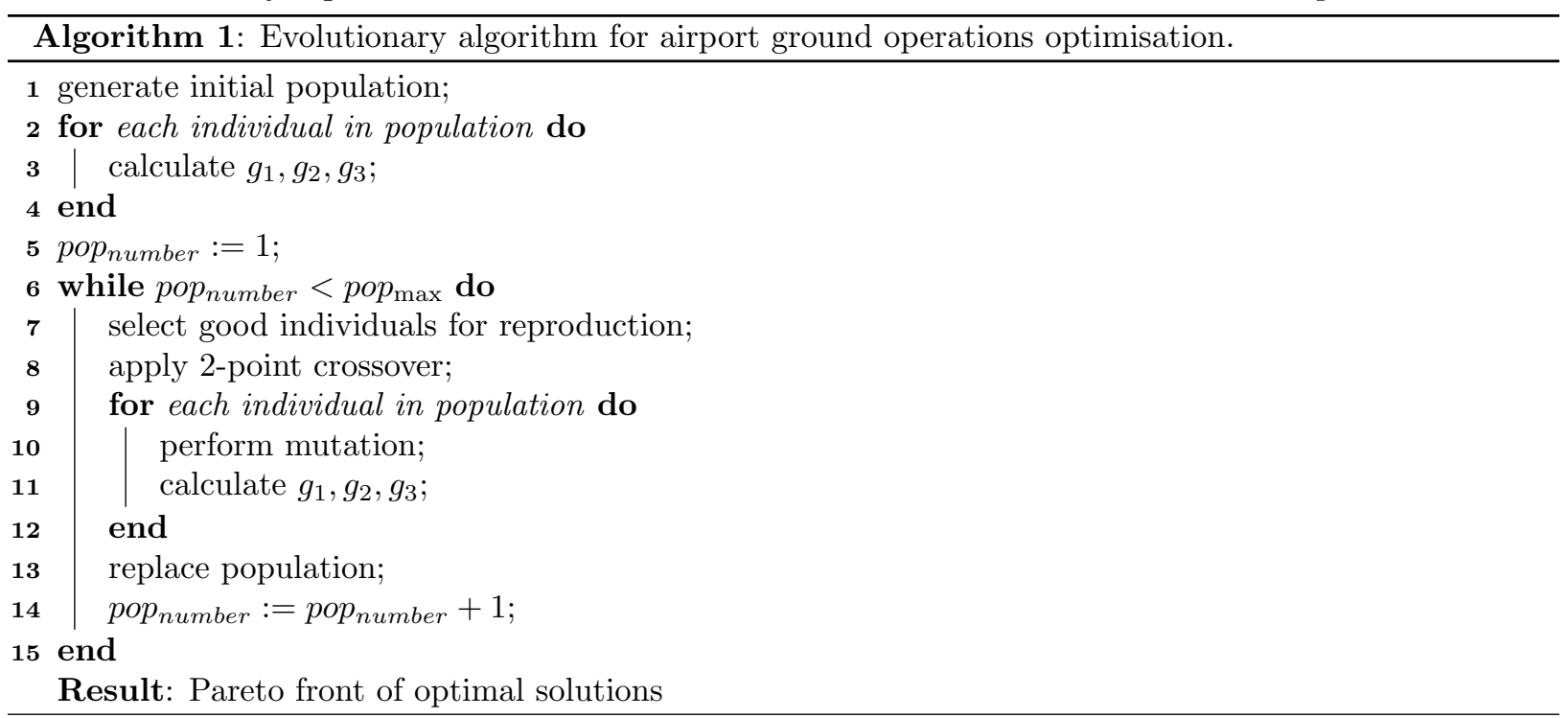

The procedure to calculate $g_{1}, g_{2}, g_{3}$ in lines 3 and 11 is further explained in detail in Algorithm 2. Firstly, aircraft are considered sequentially according to their initial sequence specified by input flight schedule. For each arriving aircraft $i \in A$ a shortest route $q_{i}$ is found between the runway and designated stand $s_{i}$ or in the opposite direction for departing aircraft $i \in D$. The generated route $q_{i}$ and weight category $v_{i}$ of aircraft $i$ is used to read the optimal speed profiles from the database. The speed profile specified by $y_{i}$ which is selected in line 8 is used to schedule the aircraft $i$ along the route $q_{i}$ after all taxiing conflicts has been resolved in line 9. Then, the total taxi time $t^{\text {taxi }}$ and the total fuel $f^{t a x i}$ is computed as stated in Section 2.2. Next, for each departing aircraft $i \in D$, the runway holding point arrival time $d_{i}$ is determined, given the scheduling of taxiing aircraft assigned during ground movement phase in lines 1-12. Subsequently, the delay $w_{i}$ is 
calculated in line 15 in order to get the total runway delay $t^{r w y}$ and the total fuel $f^{r w y}$ in line 17 . Given the pushback time $x_{i}$ from the stand $s_{i}$ for departing aircraft $i \in D$ and arrival time $z_{i}$ to the stand $s_{i}$ for arriving aircraft $i \in A$, a set of bus trip is generated in line 19 as explained in Section 2.3. By solving the corresponding VSP, the third objective $g_{3}$ can be calculated. Finally, objectives $g_{1}, g_{2}, g_{3}$ are returned in line 21 .

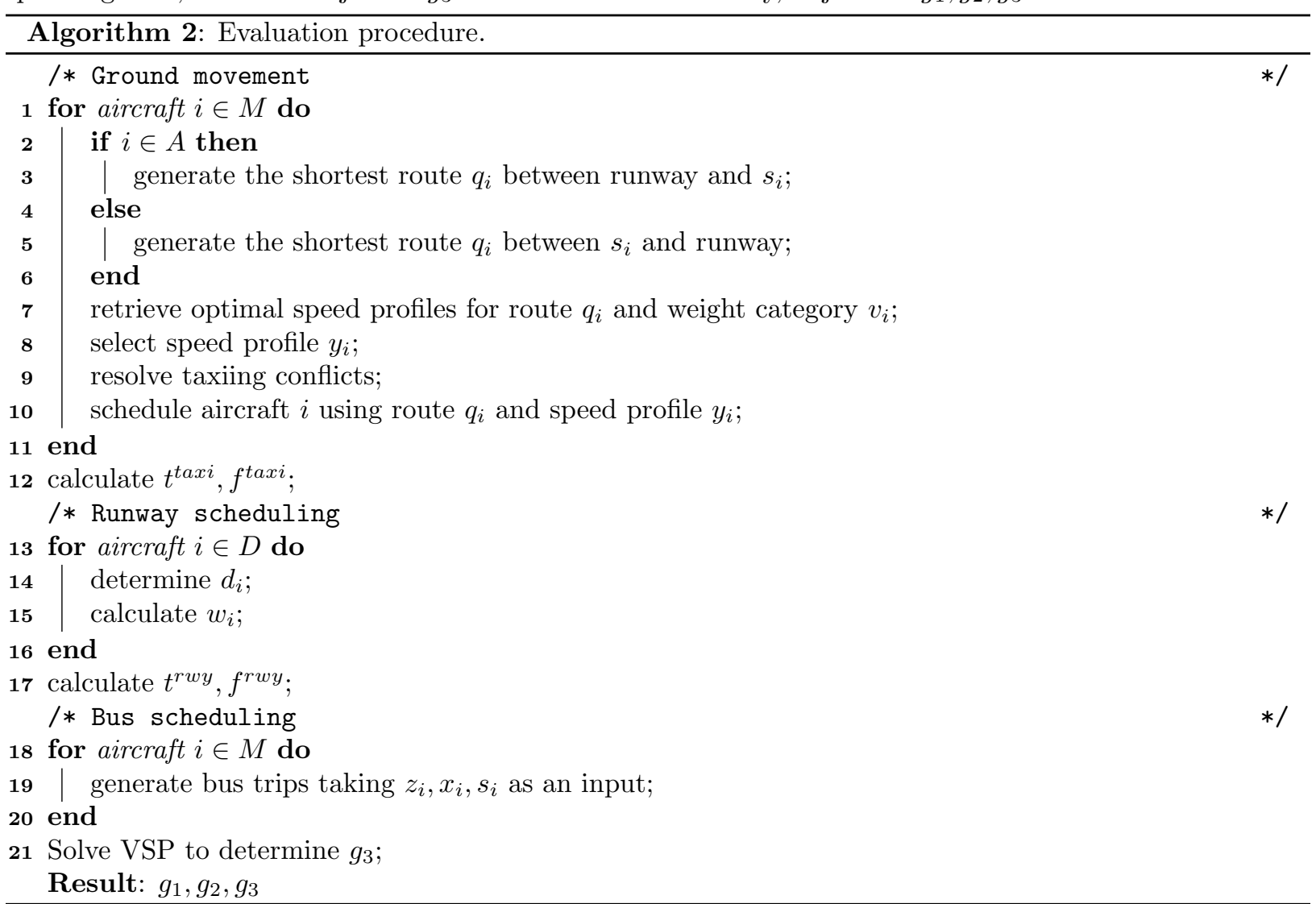

In the case of a real decision support system, the decision maker is responsible for choosing one solution found by the multi-objective integrated framework. The solutions on the Pareto front are optimal in the Pareto sense, meaning that any one of the solutions is not the best in all objectives comparing to the other one. As a result, other information is required for the decision makers in order to decide the best schedule. The next section proposes an economic framework for this purpose.

\subsection{Economical optimisation}

The conceptual framework presented in this section paves the way to a holistic (technical / environmental / economic) optimisation of the airport ground operations performance by managing how the aircraft schedules are planned. As stated in Section 2.4, the integrated optimisation problem minimises 3 objectives, namely $g_{1}, g_{2}, g_{3}$. From the economic point of view, the first two objectives $g_{1}, g_{2}$ are related to cost relevant for airline company operating the aircraft. The third objective $g_{3}$ is relevant for the airport operating the buses and it is airport dependent since large airports are generally more technically efficient and have less operational wastage than small airports [50]. The proposed economic model focuses on the cost for the airline company and therefore economic optimisation of aircraft schedules is performed from their point of view. The third objective $g_{3}$ is considered later in Section 4.3.

To be consistent with Eurocontrol [51], the following categories of aircraft strategic costs (i.e. variable or marginal costs) are relevant for aircraft ground operations:

- Fuel, as previously modelled and discussed in Section 2.2. 
- Non-fuel aircraft cost, including:

- Maintenance: they include costs of delay incurred by aircraft and related to factors such as the mechanical attrition of aircraft waiting at gates or taxiing.

- Fleet: these costs refer to the full cost of fleet financing, such as depreciation or rentals of aircraft, etc.

- Crew cost: is the variable cost of the crew personnel, i.e. pilot and flight attendant salaries.

Those non-fuel aircraft costs are specific for aircraft, and an example for an Airbus A320 aircraft according to Eurocontrol [51] is given in Table 3.

Table 3: Non-fuel aircraft costs according to [51] for base scenario for Airbus A320 aircraft.

\begin{tabular}{lcc} 
& $€ \cdot \mathrm{h}^{-1}$ & $€ \cdot \mathrm{s}^{-1}$ \\
\hline Maintenance & 720 & 0.200 \\
Fleet & 610 & 0.169 \\
Crew & 360 & 0.100 \\
Total $c^{a}$ & 1690 & 0.469 \\
\hline
\end{tabular}

The fuel cost $c^{\text {fuel }}$ of $0.71 € \cdot \mathrm{kg}^{-1}$ (as on 17.1.2014 [52]) is used in the economic optimisation model. The key idea of the proposed economic optimisation model is to sum the aircraft cost for each schedule to calculate its total aircraft strategic cost $C^{\text {total }}$ as given in Equation 13:

$$
C^{\text {total }}=c^{a} \cdot g_{1}+c^{\text {fuel }} \cdot g_{2}
$$

Knowing the cost for each schedule, it will be possible to determine the optimal schedule/group of schedules for aircraft. This is intended not to replace the human decision maker in selecting the best solution rather than provide extra information from the economic perspective.

Fig. 5 provides a qualitative idea of this approach. For solutions on the Pareto front, shorter the total time, higher the fuel cost as fast taxiing involves heavy acceleration. Similarly, lower total time results in lower aircraft cost due to their time dependency. On the other hand, too long total time causes excessive fuel burn. For each solution, by summing up the non-fuel aircraft cost and fuel cost it is possible to obtain a parabolic-like total aircraft strategic cost function with a minimum. The minimum represents the optimal or most cost effective solution among those on the Pareto front with consideration of the consumed fuel.

The next section presents results obtained by applying the proposed multi-objective framework incorporating the economic optimisation model to a real-world instance.

\section{Computational results and discussion}

\subsection{Experimental setup}

The algorithm was tested on a dataset of real arrival and departure flights on Doha International Airport $(\mathrm{DOH})$ which was the largest airport in Qatar and a hub airport for Qatar Airways until the new Hamad International airport was completed in late April 2014. DOH airport has 55 stands and no gates. The considered data was recorded on 16th March 2014 and divided into two instances representing medium and high traffic conditions. The instance medium includes 96 flights between 17:00 and 21:00 UTC from which 50 are arrivals and 46 departures. The instance high consists of 84 flights between 21:00 and 23:00 UTC from which there are 27 arrivals and 57 departures. The data provided specified landing/pushback times and gates/runway exits for each flight.

The aircraft have been divided into 3 groups according their wake vortex separation requirements. For each category, a representative aircraft is designated and its specifications are used during the fuel consumption calculation. The specifications are summarized in Table 4. 


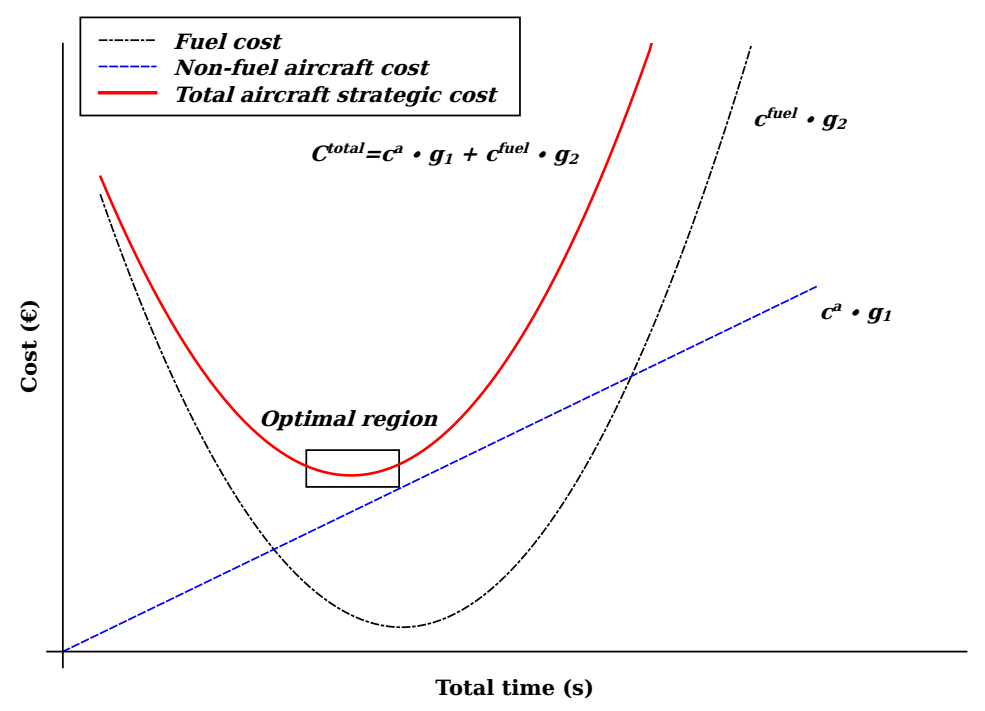

Figure 5: The total aircraft strategic cost is a sum of non-fuel aircraft cost and fuel cost.

Table 4: Specifications of the representative aircraft.

\begin{tabular}{|c|c|c|c|}
\hline & et $35 \mathrm{~A}$ & 4320 & \\
\hline Tak & $8300 \mathrm{~kg}$ & $\mathrm{~kg}$ & $00 \mathrm{~kg}$ \\
\hline & $2 \mathrm{~B}$ & $\mathrm{CMF}$ & CF6- \\
\hline & & & \\
\hline & $\mathrm{N}$ & T & \\
\hline$F$ & & & \\
\hline el & $0.024 \mathrm{~kg} \cdot \mathrm{s}^{-1}$ & $0.101 \mathrm{~kg} \cdot \mathrm{s}^{-1}$ & $0.228 \mathrm{~kg} \cdot \mathrm{s}^{-}$ \\
\hline uel flow at $30 \% R$ & $0.067 \mathrm{~kg} \cdot \mathrm{s}^{-1}$ & $0.291 \mathrm{~kg} \cdot \mathrm{s}^{-1}$ & $0.724 \mathrm{~kg}$. \\
\hline
\end{tabular}

The computational experiments were performed on a computer with an Intel i3-2120 processor and 3.16 GB of RAM, running Linux. The evolutionary algorithm is implemented using the Inspyred package for Python [53]. Based on initial experiments, the maximum number of generations pop $p_{\max }$ was set to 150 and each population contained 200 individuals.

\subsection{Sensitivity analysis of bus scheduling}

Before the actual computational experiments, a sensitivity analysis of bus scheduling was performed to investigate the effect of different time parameters set in Section 2.3. Namely, arrival time of the buses after arriving aircraft came to a stand $p_{a r r}$, embarking/disembarking time of passengers $p_{e m b}$, headway between buses $p_{\text {headway }}$, departure time of buses before the pushback time of departing aircraft $p_{\text {dep }}$ and average speed of buses sp. The parameters were varied, one at a time, from the established base case, as set in Section 2.3, over a reasonable range. Then, Algorithm 1 with objective $g_{3}$ was run to find a solution with minimum bus scheduling cost under current parameters. The impact of parameters on overall bus scheduling cost is shown in Fig. 7. As can be seen, embarking/disembarking time of passengers $p_{\text {emb }}$ and average speed of buses $s p$ have the most influence on bus scheduling cost with approximately linear relationship for $p_{e m b}$ and inversely proportional relationship for $s p$. In contrast, arrival and departure time of buses $p_{a r r}, p_{d e p}$ or headway $p_{\text {headway }}$ had only little effect on bus scheduling cost. In conclusion, setting the appropriate bus scheduling parameters accurately, namely embarking/disembarking time of passengers $p_{\text {emb }}$ and average 


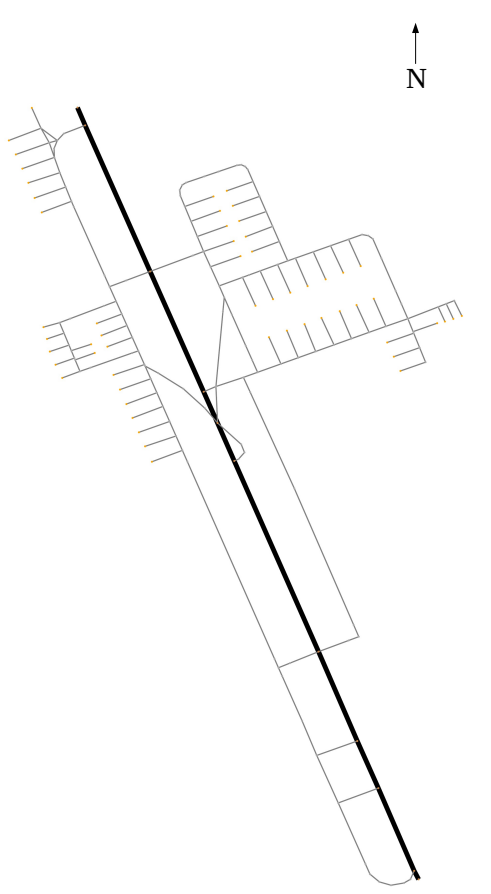

(a)

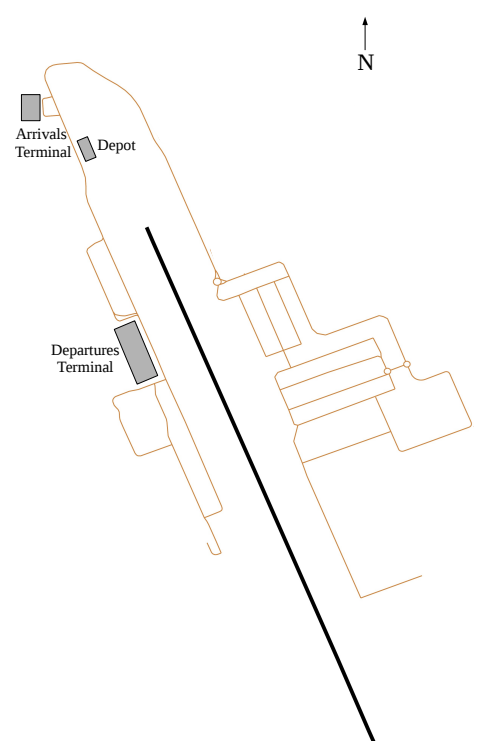

(b)

Figure 6: Layout of DOH airport with taxiways (a) and service road network (b) used by buses.

speed of buses $s p$, is an important task for airport management. However, as their effect is approximately proportional, they do not affect the generalisation of results obtained by computational experiments carried out in next sections.

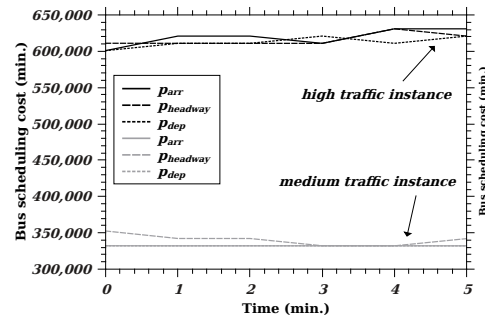

(a)

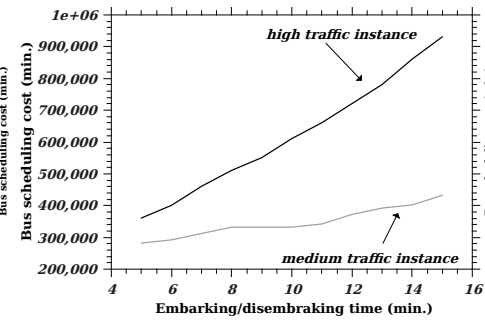

(b)

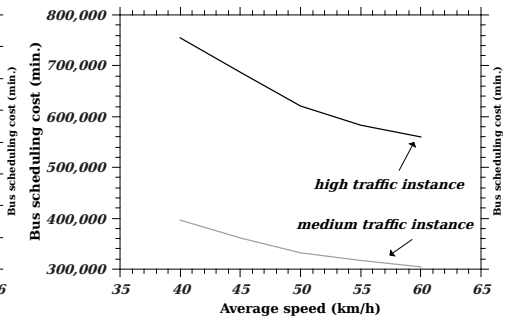

(c)

Figure 7: Sensitivity analysis of bus scheduling: Minimum bus scheduling cost obtained with varying parameters (a) arrival time $\left(p_{\text {arr }}\right)$, departure time $\left(p_{\text {dep }}\right)$ and headway $\left(p_{\text {headway }}\right)$ of buses, $(\mathrm{b})$ embarking/disembarking time of passengers $\left(p_{e m b}\right)$, and (c) average speed of buses $(s p)$.

\subsection{Comparison of different approaches}

As reviewed in Section 1, previous research usually treated the ground movement problem in isolation from other surface operations as well as focused only on one objective. Therefore, we start our results by comparison of our integrated approach with previous approaches and present the benefit of the proposed method. Section 2.4 stated that three objectives $g_{1}, g_{2}, g_{3}$ related to total time, fuel consumption and bus scheduling are considered in this paper. In order to simulate different approaches previously proposed in 
research literature, we introduce weights $h_{1}, h_{2}, h_{3}, h_{4}$ with possible values $\{0,1\}$ in Equation 14 , which enable us to "switch off" various part of the objective function. Using different combinations of weights as shown in Table 5, the following algorithms have been devised according to the previously proposed approaches as reviewed in Section 1. It is worth mentioning that the implemented approaches in Table 5 are all based on the optimised speed profiles to calculate their respective fuel consumption. Therefore, results presented in the following sections have been improved in terms of fuel compared to their counterparts in the original literature. Algorithms A and B correspond to approach in which only ground movement or runway scheduling is being optimised, taking into account only the total taxi time or total runway delay, respectively. Algorithm $\mathrm{C}$ represents the integrated ground movement and runway scheduling optimisation with only time objective $g_{1}$ minimised. Ground movement optimisation with total taxi time and taxi fuel minimisation is considered in Algorithm D. Integrated runway scheduling and ground movement with time and fuel objective is minimised in Algorithm E. Finally, Algorithm I corresponds to approach proposed in this paper, with fully integrated runway scheduling, ground movement and bus scheduling optimisation and $g_{1}, g_{2}, g_{3}$ objectives.

$$
\begin{aligned}
& g_{1}=h_{1} \cdot t^{\text {taxi }}+h_{2} \cdot t^{r w y} \\
& g_{2}=h_{3} \cdot\left(f^{\text {taxi }}+f^{\text {rwy }}\right) \\
& g_{3}=h_{4} \cdot g_{3}
\end{aligned}
$$

\begin{tabular}{|c|c|c|c|c|c|c|}
\hline $\begin{array}{l}\text { Weight } \\
\text { Alg. }\end{array}$ & $\begin{array}{c}h_{1} \\
t^{\operatorname{tax} i}\end{array}$ & $\begin{array}{c}h_{2} \\
t^{r w y}\end{array}$ & $\begin{array}{l}h_{3} \\
g_{2}\end{array}$ & $\begin{array}{l}h_{4} \\
g_{3}\end{array}$ & Description & References \\
\hline $\mathrm{A}$ & 1 & 0 & 0 & 0 & Only taxi time & $\begin{array}{l}{[15,16,17,18,19} \\
20,21,22]\end{array}$ \\
\hline B & 0 & 1 & 0 & 0 & Only runway delay & {$[8,9,10,11,12]$} \\
\hline $\mathrm{C}$ & 1 & 1 & 0 & 0 & $\begin{array}{l}\text { Integrated runway scheduling } \\
\text { and ground movement, only time } \\
\text { objective }\end{array}$ & {$[29,30,31,32]$} \\
\hline $\mathrm{D}$ & 1 & 0 & 1 & 0 & Ground movement with fuel & $\begin{array}{l}{[23,24,25,26,27,} \\
28]\end{array}$ \\
\hline $\mathrm{E}$ & 1 & 1 & 1 & 0 & $\begin{array}{l}\text { Integrated runway scheduling } \\
\text { and ground movement, time and } \\
\text { fuel objective }\end{array}$ & This paper \\
\hline $\mathrm{I}$ & 1 & 1 & 1 & 1 & $\begin{array}{l}\text { Integrated runway scheduling, } \\
\text { ground movement and bus } \\
\text { scheduling }\end{array}$ & This paper \\
\hline
\end{tabular}

Table 5: Alternative algorithms devised by considering objective functions with different weights corresponding to research approaches reviewed in Section 1.

Note, that although some algorithms do not consider ground movement, the solutions generated by these algorithms use the optimised speed profiles as explained in Section 2.2.

\subsubsection{Visual comparison}

Algorithm 1 was run with objective functions as given in Equation 14 with weights set according to Table 5. The Pareto front for each Algorithm A-I was constructed by considering 30 repeated runs of the algorithm and leaving only non-dominated solutions. The fronts are depicted in Fig. 8. In order to provide a better overview of the results, the fronts are projected to 2-objective space in Fig. 9 and 10, considering 


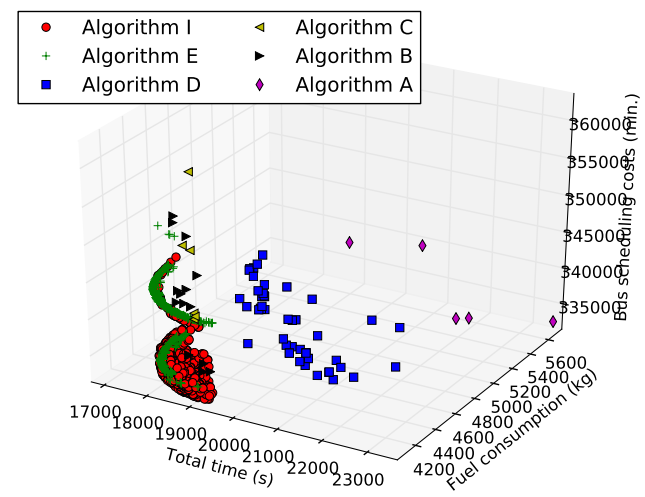

(a)

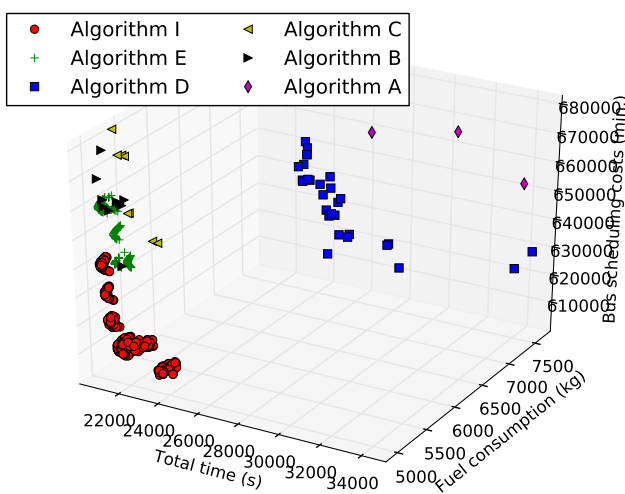

(b)

Figure 8: Pareto fronts obtained by different algorithms for (a) medium traffic instance and (b) and high traffic instance.

$g_{1}, g_{2}, g_{1}, g_{3}$ and $g_{2}, g_{3}$, respectively. By visually examining the Pareto fronts, it can be observed that the proposed algorithms performed similarly for both data instances.

With regard to the total time objective $g_{1}$, it can be observed, that Algorithm A and D resulted in solutions with a very high value of $g_{1}$. Minimisation of objective $g_{1}$ was the main aim of Algorithm C. As a result, it obtained the best values of the total time $g_{1}$.

For the fuel consumption (objective $g_{2}$ ), Algorithm A,C and D obtained higher values than other algorithms. The algorithm that performs the best in terms of $g_{2}$ cannot be determined by visual observation.

Considering the bus scheduling cost objective $g_{3}$, it can be seen, that for the medium traffic instance Algorithms A-I performed similarly. In contrast, for the high traffic instance, Algorithm I obtained the minimum values of $g_{3}$.

From the visual observation, it can be concluded that Algorithm I and E resulted in the best trade-off curves compared to other algorithms. Note, that in case of high traffic instance the evolutionary algorithm produced the front with worse spread for Algorithm I compared to Algorithm E as can be seen in Fig. 10 due to increased complexity of the instance. Otherwise, Algorithm I covered most of the Pareto optimal solutions generated by Algorithm E.

Finally, it can be observed that inclusion of all three objectives $g_{1}, g_{2}, g_{3}$ increased the number of nondominated solution found by Algorithm I. This result can be beneficial in case of unprecedented events, when the decision maker needs to have more readily available alternatives as backup plans. More solutions then enable the decision maker to select another schedule without sacrificing too much of objective $g_{1}, g_{2}$ or $g_{3}$. In order to illustrate the effect of unprecedented situation, the following example is presented. Lets assume, that the decision maker selected a solution for the high traffic instance with $\left[g_{1}, g_{2}, g_{3}\right]=$ $[21411,5030,620914]$ in which departing aircraft \#127 has a scheduled pushback time 21:56:47. Then, if a delay of 3 minutes occurs, schedule has to be modified. There is another solution in the Pareto front with $\left[g_{1}, g_{2}, g_{3}\right]=[21441,5025,620906]$ in which aircraft preceding \#127 have the same values of decision variables as in the original solution, but \#127 has pushback time 22:00:58. The schedule can be modified by implementing the rest of the new solution, resulting in $\left[g_{1}, g_{2}, g_{3}\right]=[21445,5025,620906]$. As can be seen, the new solution is similar in terms of $g_{1}, g_{2}, g_{3}$ to the originally selected solution. Note, that the presence of an alternative solution is not guaranteed by the algorithm and the ability to provide robust solutions will be investigated in future work.

As intuitive results shown above, it seems that the introduced multi-objective optimisation framework so 


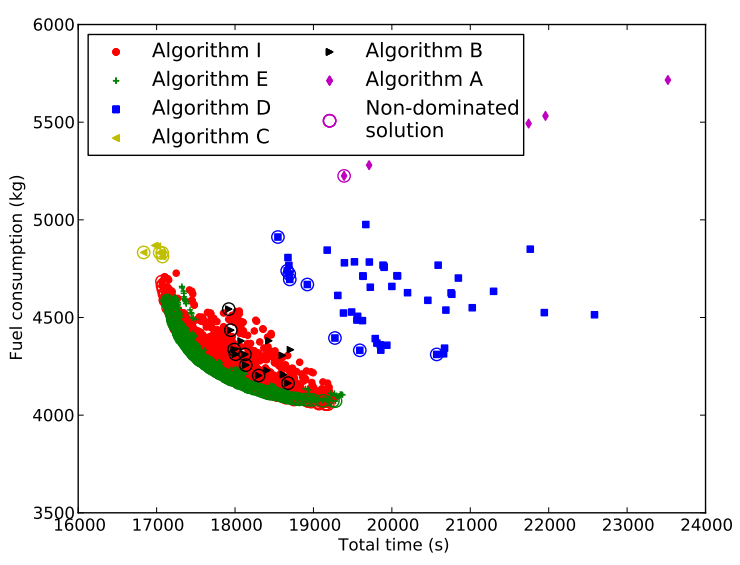

(a)

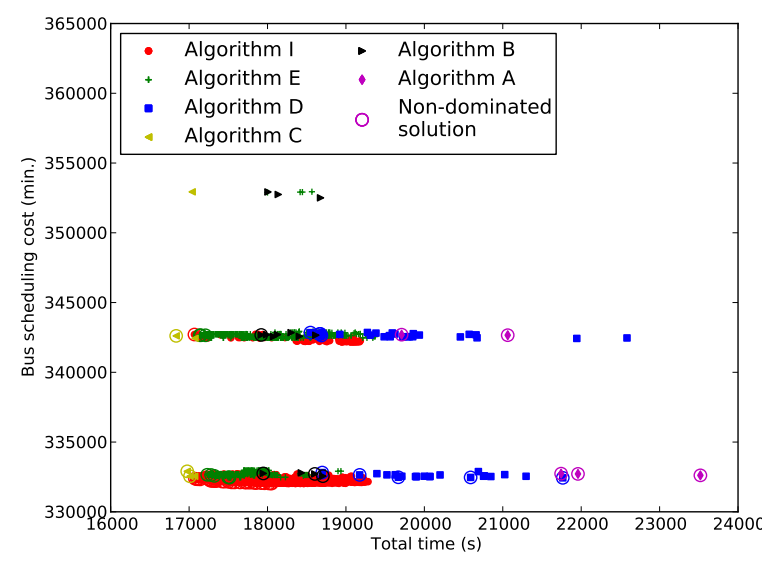

(b)

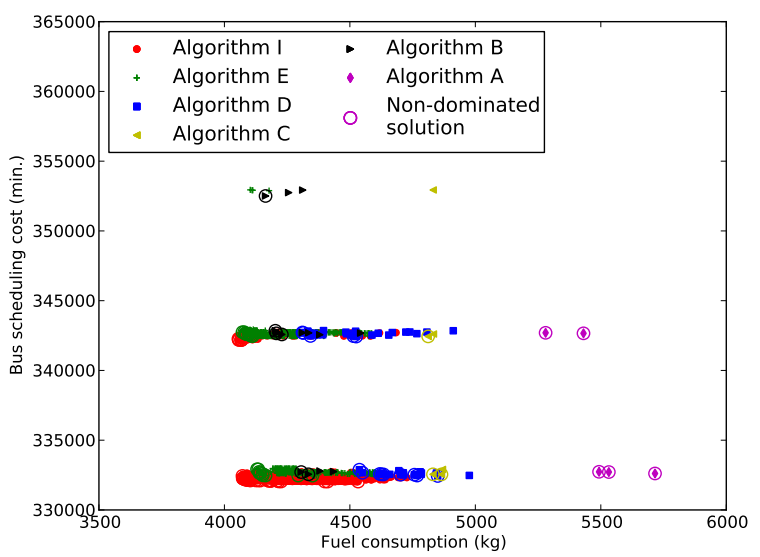

(c)

Figure 9: Pareto fronts obtained by different algorithms for medium traffic instance projected to (a) $g_{1}, g_{2}$, (b) $g_{1}, g_{3}$ and (c) $g_{2}, g_{3}$.

far opens a door for decision makers to make a more reasonable planning, and also provides more evidence and information to back such decisions. However, it is still the case that without additional information, the decision process is still very subjective based as multiple Pareto optimal solutions are available. This situation will be even more severe due to visualisation difficulty as the number of the investigated objectives increases. Furthermore, in terms of the performances of different algorithms, without a single measure, it is hard to be convinced which algorithm performs the best. Therefore, in the next section, the proposed economic optimisation in Section 3.2 is applied once the Pareto solutions from the multi-objective framework are obtained.

\subsubsection{Economic results}

This section gives an account of the economic results. By applying the economic optimisation model as explained in Section 3.2, it is possible to determine the monetary value of the total time $g_{1}$ and the fuel burned $g_{2}$ and obtain a single optimal solution (or, more broadly speaking an optimal region of the Pareto front) from the economic point of view. For this purpose, the costs stated in Table 3 for Airbus A320 and 


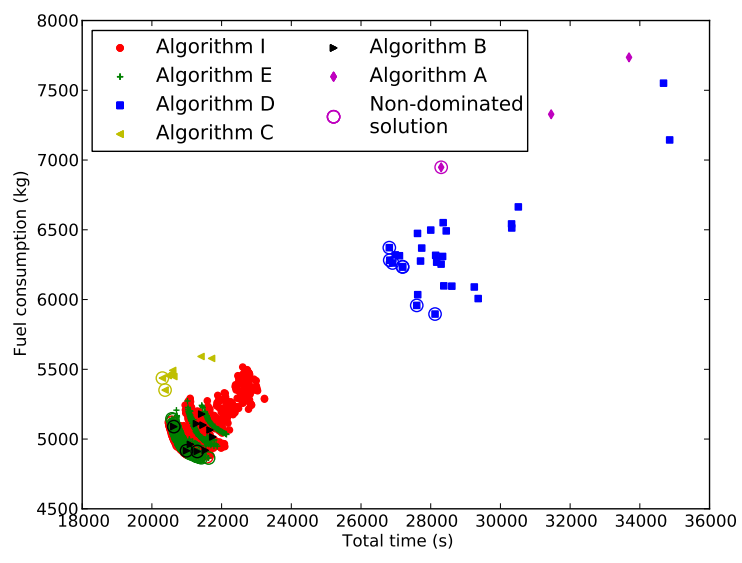

(a)

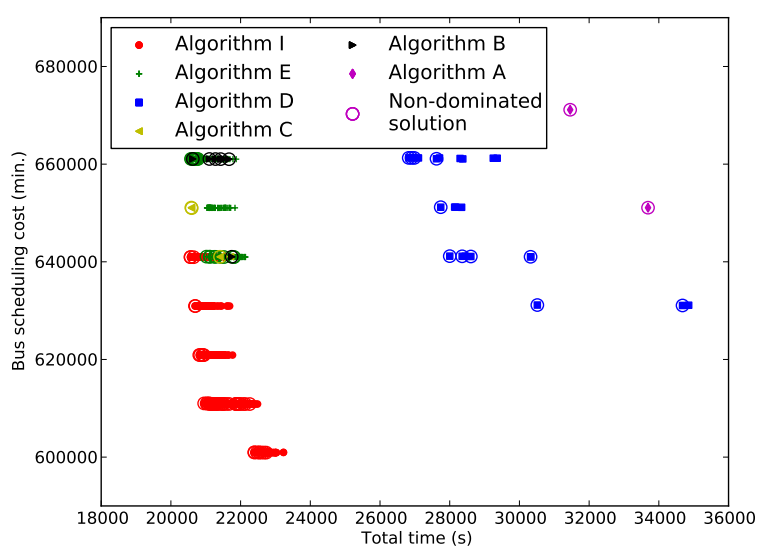

(c)

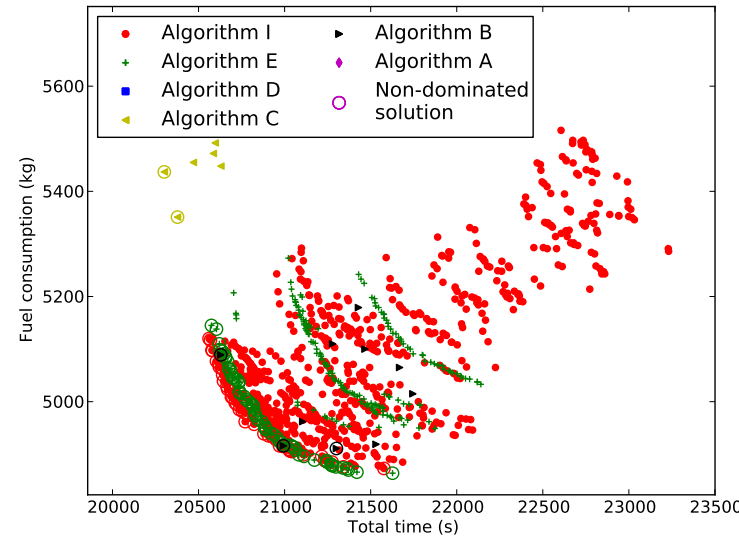

(b)

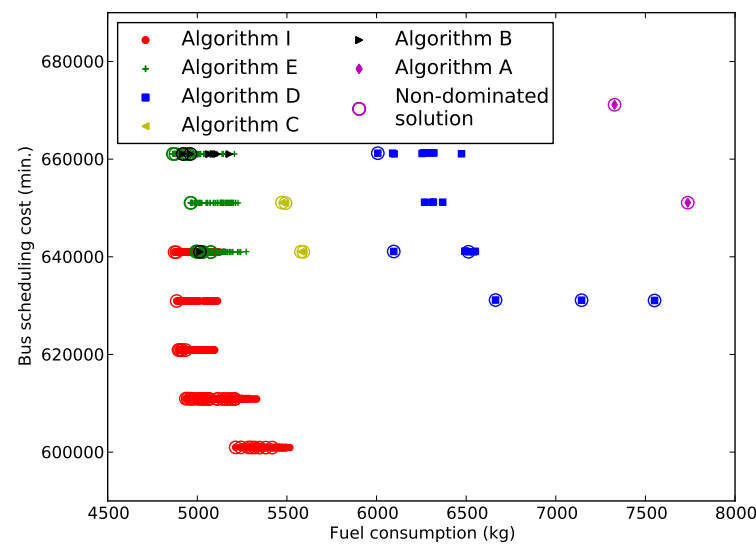

(d)

Figure 10: Pareto fronts obtained by different algorithms for high traffic instance projected to (a) $g_{1}, g_{2}$ in a global view, (b) $g_{1}, g_{2}$ in a zoomed view, (c) $g_{1}, g_{3}$ and (d) $g_{2}, g_{3}$.

the fuel price are used as an example. Firstly, the value of the corresponding objective is multiplied by the total non-fuel aircraft cost and fuel price according to Equation 13. Fig. 11 and 12 depict the Pareto front after the modification. Then, the solution with the minimum costs can be selected for each algorithm. In order to consider statistical inference, the solution with minimum costs was selected from the Pareto front of each of 30 runs of the algorithm. Table 6 summarises the average values of optimal solutions of different algorithms for each data instance.

The comparison of algorithm performance supports the observations described in Section 4.3.1. The best values of the total time objective $g_{1}$ are obtained by Algorithm $\mathrm{C}$ in both data instances by adding up low values of both total taxi time $t^{\text {taxi }}$ and runway delay $t^{r w y}$. However, the best values of the total time $g_{1}$, are only possible at the expense of fuel intensive speed profiles, causing a high value of $g_{2}$. Algorithm A resulted in the worst solutions related to the total time (objective $g_{1}$ ). Since Algorithm A minimised only the total taxi time $t^{t a x i}$, it did not consider the runway delay. The additional waiting at the runway 


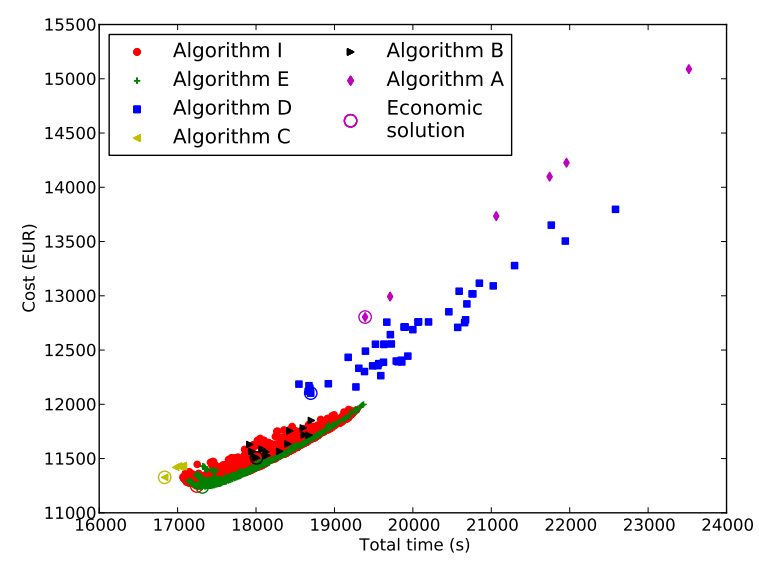

(a)

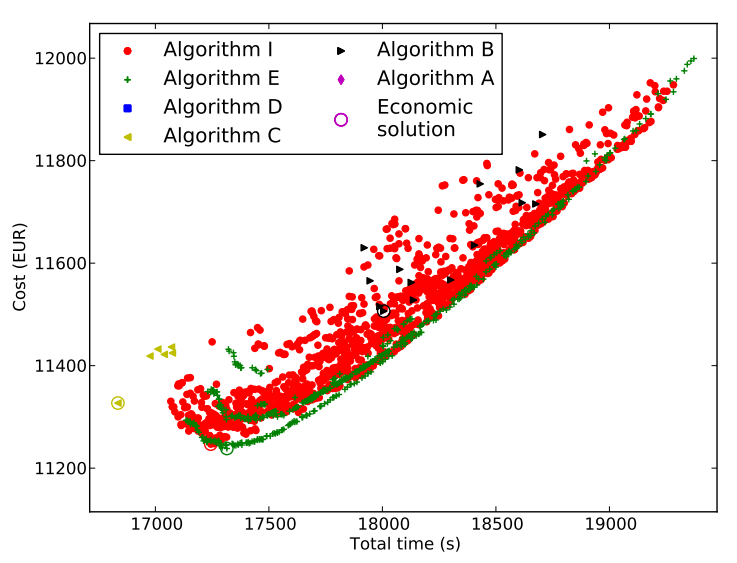

(b)

Figure 11: Economic analysis of Pareto fronts for medium traffic instance: (a) a global view, (b) a zoomed in view. Circles indicate the economic solution for the given algorithm.

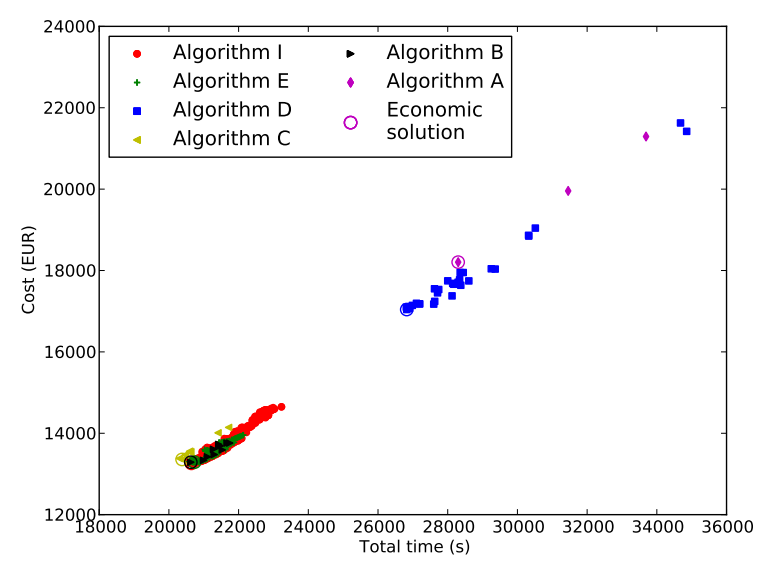

(a)

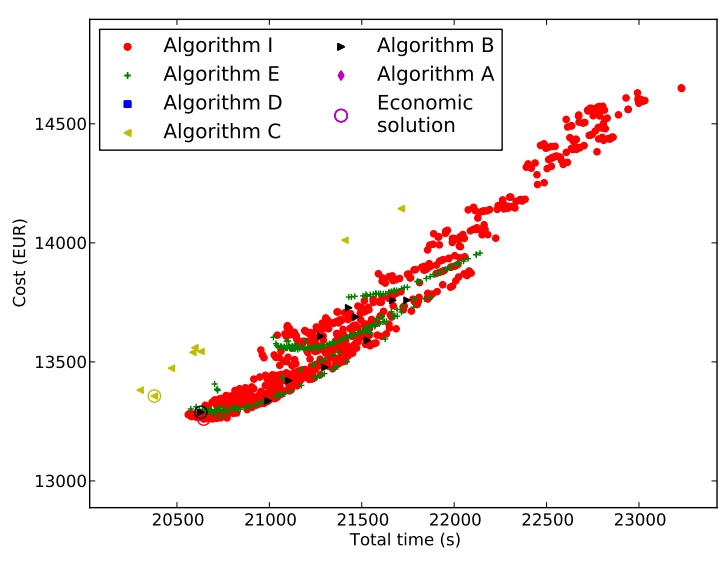

(b)

Figure 12: Economic analysis of Pareto fronts for high traffic instance: (a) a global view, (b) a zoomed in view. Circles indicate the economic solution for the given algorithm.

(runway delay $t^{r w y}$ ) then increased the total time $g_{1}$. Compared to the solution with the minimum cost, Algorithm A required $25 \%$ and $46 \%$ more fuel for medium and high traffic instances, respectively. For the fuel consumption objective $g_{2}$, the best solutions selected by economic optimisation are those obtained by Algorithm B for the both traffic instances. In general, algorithms that included runway scheduling, or runway scheduling and fuel consumption, i.e. Algorithm B, E and I, found solutions with the best values of $g_{2}$. In the case of Algorithm B, the low value of $f^{r w y}$ is caused by optimised $t^{r w y}$ and the low value of $f^{t a x i}$ is a result of high total taxi time $t^{\text {taxi }}$. As explained in Section 2.2, there is a trade-off between $t^{\text {taxi }}$ and $f^{t a x i}$, which means that the value of one cannot be decreased without increasing the value of another. All other algorithms (Algorithm A,C,D) which do not considered runway scheduling, have their $g_{2}$ increased by additional fuel burn at the runway (Algorithm A,D) or very short taxi times requiring fuel intensive speed 
Table 6: The average values of optimal solutions obtained by economic optimisation for each instance with the overall best value highlighted in bold face.

\begin{tabular}{ccccccccc} 
Algorithm & $t^{\text {taxi }}(\mathrm{s})$ & $f^{\text {taxi }}(\mathrm{kg})$ & $t^{\text {rwy }}(\mathrm{s})$ & $f^{\text {rwy }}(\mathrm{kg})$ & $g_{1}(\mathrm{~s})$ & $g_{2}(\mathrm{~kg})$ & $g_{3}(\mathrm{~min})$. & $C^{\text {total }}(€)$ \\
\hline & \multicolumn{7}{c}{ medium traffic instance } \\
\hline A & 16138 & 4768 & 5612 & 748 & 21750 & 5516 & 346460 & 14117 \\
B & 17584 & 4208 & 825 & 124 & 18409 & $\mathbf{4 3 3 2}$ & 343728 & 11709 \\
C & 16180 & 4717 & 1031 & 152 & $\mathbf{1 7 2 1 1}$ & 4869 & 341745 & 11529 \\
D & 16528 & 4316 & 3221 & 445 & 19749 & 4762 & 344084 & 12643 \\
E & 16639 & 4284 & 982 & 145 & 17621 & 4429 & 344407 & $\mathbf{1 1 4 0 8}$ \\
I & 16677 & 4329 & 907 & 133 & 17584 & 4463 & $\mathbf{3 3 6 4 0 2}$ & 11415 \\
\hline & & \multicolumn{7}{c}{ high traffic instance } \\
A & 15884 & 4935 & 16737 & 2610 & 32621 & 7545 & 680548 & 20656 \\
B & 17259 & 4388 & 4296 & 729 & 21554 & $\mathbf{5 1 1 6}$ & 665068 & 13742 \\
C & 16211 & 4733 & 4798 & 810 & $\mathbf{2 1 0 0 8}$ & 5543 & 670756 & 13789 \\
D & 16537 & 4425 & 12050 & 1914 & 28586 & 6339 & 673511 & 17908 \\
E & 16856 & 4392 & 4562 & 761 & 21419 & 5153 & 665751 & $\mathbf{1 3 7 0 4}$ \\
I & 16957 & 4400 & 4659 & 773 & 21616 & 5174 & $\mathbf{6 3 0 9 8 1}$ & 13811 \\
\hline
\end{tabular}

profiles (Algorithm C). The best solutions in terms of the third objective $g_{3}$ are those obtained by Algorithm I, which included bus scheduling costs in its objective function.

The overall results for medium and high traffic instances, as given in Table 6, show that $t^{r w y}$ and subsequently $f^{r w y}$ have much higher values in the high traffic instance compared to the medium traffic instance. This increase is due to high traffic level, causing a congestion on the runway. Similarly, higher bus scheduling costs $g_{3}$ are the result of high number of flights arriving/departing within a short time period.

In terms of costs $C^{\text {total }}$, Algorithm $\mathrm{E}$ found solutions with minimum average cost for both instances. The statistical significance of differences was further analysed by performing Student's t-test with significance level 0.05. The results of Algorithm E are not significantly better than results of Algorithm I for both instances. Algorithm E is significantly better than Algorithms A-D for the medium traffic instance and Algorithms A,C,D for high traffic instance.

Regarding the computational time, Algorithms A-E run about 8 to 10 minutes on average for the medium and high traffic instances, whereas the running time of Algorithm I was 3 times longer.

As explained in Section 3.2, the selection of a single solution from the Pareto front made by the economic optimisation is affected by $\operatorname{costs} c^{a}$ and $c^{f u e l}$. If $\operatorname{costs} c^{a}$ and $c^{f u e l}$ put more focus on the fuel objective $g_{2}$, the selected solutions will tend to have lower fuel consumption. However, only algorithms that are multiobjective, particularly Algorithms E and I, are able to produce a diverse set of trade-off solutions. Therefore, although Algorithm B obtained the lowest value of $g_{2}$ for the medium traffic instance, the difference in total cost $C^{\text {total }}$ between Algorithm B and Algorithms E,I failed to prove significant for the high traffic instance, which implies, with different $\operatorname{costs} c^{a}$ and $c^{\text {fuel }}$, it may not be able to provide solution with lower fuel consumption (and subsequently $C^{\text {total }}$ ) than solutions generated by other multi-objective algorithms. For example, with $c^{a}=0.1 € \cdot \mathrm{s}^{-1}$ and $c^{\text {fuel }}=1 € \cdot \mathrm{kg}^{-1}$, the economic optimisation selects solutions given in Table 7. In this case, analysis using Student's t-test with the same significance level 0.05 revealed that the best algorithm in terms of costs (Algorithms E and I) is significantly better than other algorithms. More importantly, results of Algorithm B in terms of fuel consumption (objective $g_{2}$ ) are significantly worse than those of Algorithm E and I.

The analysis of results from the economic point of view showed a similar pattern (supporting the reliability of the algorithms involved), and several results can be generalised. The most relevant are as follows:

- There is a real economic merit in selecting the best schedules considering the aircraft costs. 
Table 7: The average values of optimal solutions obtained by economic optimisation with $c^{a}=0.1 € \cdot \mathrm{s}^{-1}$ and $c^{\text {fuel }}=1 € \cdot \mathrm{kg}^{-1}$ for each instance with the overall best solution highlighted in bold face.

\begin{tabular}{|c|c|c|c|c|}
\hline Algorithm & $g_{1}(\mathrm{~s})$ & $g_{2}(\mathrm{~kg})$ & $g_{3}$ (min.) & $C^{\text {total }}(€)$ \\
\hline & \multicolumn{4}{|c|}{ medium traffic instance } \\
\hline $\mathrm{A}$ & 21750 & 5516 & 346460 & 7691 \\
\hline $\mathrm{B}$ & 18409 & 4332 & 343728 & 6172 \\
\hline $\mathrm{C}$ & 17211 & 4869 & 341745 & 6590 \\
\hline $\mathrm{D}$ & 20862 & 4486 & 345412 & 6572 \\
\hline $\mathrm{E}$ & 18765 & 4131 & 346747 & 6007 \\
\hline \multirow[t]{2}{*}{$\mathrm{I}$} & 18867 & 4120 & 339047 & 6007 \\
\hline & \multicolumn{4}{|c|}{ high traffic instance } \\
\hline $\mathrm{A}$ & 32621 & 7545 & 680548 & 10807 \\
\hline B & 21554 & 5116 & 665068 & 7272 \\
\hline $\mathrm{C}$ & 21008 & 5543 & 670756 & 7644 \\
\hline $\mathrm{D}$ & 29151 & 6202 & 676516 & 9117 \\
\hline $\mathrm{E}$ & 21970 & 5008 & 664748 & 7205 \\
\hline $\mathrm{I}$ & 22150 & 5042 & 633313 & 7257 \\
\hline
\end{tabular}

- The algorithms allowing to reach the minimum total aircraft cost are those with an holistic perspective considering both ground movement and runway scheduling minimising time and fuel objectives simultaneously.

- The inclusion of bus scheduling has not a significant impact on the performance in terms of economic optimisation regarding aircraft cost. From an airline only perspective, Algorithm E and I are equivalent without significant differences in costs.

- The difference between the most cost efficient solution for Algorithm E and I in Table 6 is up to around $5 \%$ in bus scheduling cost. This is a saving relevant from the airport operator's point of view.

- Economical optimisation can be used for different scenarios (high or low non-fuel and fuel cost) as shown in Table 6 and 7 . As fuel price is taken into account, it always takes environmental benefit into consideration.

In conclusion, Algorithm I can be considered as the best one since it allows an optimisation from both the perspectives of the airline and the airport operator.

\section{Conclusions and Future development}

This paper introduced an integrated optimisation approach to airport ground operations. A multiobjective genetic algorithm which considers several elements: ground movement problem, runway scheduling and scheduling of airport buses was proposed to find aircraft schedules in a more holistic manner through actively incorporating multi-objective 4DTs and economic optimisation. The integrated systems approach facilitates more detailed investigation of the trade-off between different objectives and furthermore, the most cost-effective solution, taking into account fuel saving, from a Pareto front of optimal solutions can be obtained through the economic optimisation framework which assigns a monetary value to the given schedule. The computational experiments conducted on real-world data from a major Asian airport showed that the proposed approach is able to provide a systematic optimisation framework suitable for decision support at the airport. Such decision support serves to achieve the aim of A-CDM concept to optimise airport-related decisions in collaboration with both airline and airport operator, considering their preferences and constraints utilising shared information. A detailed comparison of algorithms with different objective functions, corresponding to previously applied approaches reviewed in Section 1 and categorized in Table 5, 
showed that the integrated approach results in solutions with the lowest costs. Furthermore, the integrated approach obtained better results in terms of fuel consumption (19\% and $31 \%$ less fuel burned for the economical solution compared to the worst solution). Bear in mind, algorithms mentioned in this paper used optimised 4DTs already. Therefore, the proposed approach not only saves fuel by using 4DTs, but also a further reduction is achieved by integrated multi-objective optimisation framework. Only the approach which considered bus scheduling (Algorithm I) could find a schedule with low bus scheduling cost, particularly in the high traffic period. To conclude, previously proposed approaches (represented by Algorithms A,B,D) that considered ground movement problem, runway scheduling and ground services scheduling as isolated problems, or optimised only time objective (Algorithm C), performed worse than the integrated approaches (Algorithms E and I). The result indicates that there are strong interdependencies between airport ground operations and respective time, fuel, and cost related objectives which were not fully taken into account in previously proposed approaches. As the proposed optimisation approach which actively promotes integrated and coordinated airport ground operations is better in terms of performance, economic and environmental criteria, it can contribute to sustainability of air transportation. Furthermore, only the integrated approach can take into account objectives of the airline and the airport at the same time.

The generality of the proposed integrated optimisation approach is supported by the fact that it is not dependent on the layout of a particular airport, nor cost values, making it an approach applicable to other airports as well. However, the most benefit yielded by its application can be obtained on large and busy airports, where runway needs to be used as efficiently as possible, with high traffic on airport surface and high requirement of ground services for the aircraft, such as Doha International airport used as a case study in this paper. Furthermore, airports that face strict environmental regulations, putting more focus on fuel consumption, may find the proposed approach beneficial.

Nevertheless, a number of limitations of the proposed approach need to be mentioned and further adressed in the future research. First, uncertainty inherent in airport operations has to be considered in order to make the approach realistic. Delayed arrivals due to weather conditions, aircraft taxiing slower/faster than prescribed by 4DTs or baggage being loaded late into the hold are only some examples of uncertainty that can compromise otherwise optimal planning. Furthermore, ever changing situation at an airport calls for a real-time scheduling which together with uncertainty could be tackled by dynamic and robust optimisation techniques in the future. Other limitations open for further investigation include the application of a more sophisticated routing algorithm for ground movement such as $k$-QPPTW [26], or more precise setting of bus scheduling related parameters such as embarking/disembarking time based on real number of passengers in each flight.

Finally, a number of further developments are possible from the economic point of view. Along with the aircraft cost there are other costs that are possible to include, namely airport cost and externalities. Every minute during which the airport infrastructure is used in an inefficient way is a cost for the airport, particularly during the peak traffic period. Since different periods during the day have different demand (peak vs. off-peak), airport cost may change over the day. Moreover this cost depends on the airport: some airports are very busy, other underused. Therefore, time objective can be priced to reflect cost for the airport as well.

Furthermore, further works should be devoted to the quantification and inclusion of the externalities. Externalities are costs or benefits that affect a party who did not choose to incur those costs or benefits. In the case considered, the key externalities are air emissions (in particular $\mathrm{CO}_{2}$ and $\mathrm{NO}_{\mathrm{x}}$ ) and noise. It is possible to assign to them a monetary value and include them in the analysis.

\section{Acknowledgements}

This work is supported in part by the Engineering and Physical Sciences Research Council (EPSRC) under Grant EP/H004424/1. The authors would also like to thank Mohammed Ali who provided the real dataset. 


\section{References}

[1] ICAO, . Annual Report of the ICAO Council: 2013 The World of Air Transport.

2014.

URL http://www.icao.int/annual-report-2013/Pages/the-world-of-air-transport-in-2013.aspx.

[2] European Commission, . Flightpath 2050: Europe's Vision for Aviation. http://ec. europa.eu/transport/modes/air/doc/flightpath2050.pdf.

[3] Liem, R.P., Kenway, G.K., Martins, J.R.. Multimission Aircraft Fuel-Burn Minimization via Multipoint Aerostructural Optimization. AIAA Journal 2014;53(1):104-122.

[4] Takeshita, T.. Competitiveness, role, and impact of microalgal biodiesel in the global energy future. Applied Energy 2011;88(10):3481-3491.

[5] Chuck, C.J., Donnelly, J.. The compatibility of potential bioderived fuels with Jet A-1 aviation kerosene. Applied Energy 2014;118:83-91.

[6] Trivedi, P., Olcay, H., Staples, M.D., Withers, M.R., Malina, R., Barrett, S.R.. Energy return on investment for alternative jet fuels. Applied Energy 2015;141:167-174.

[7] Eurocontrol, . Airport CDM Implementation Manual. 2012. URL http://www.eurocontrol.int/sites/default/files/publication/files/

[8] Balakrishnan, H., Chandran, B.. Scheduling aircraft landings under constrained position shifting. In: AIAA Guidance, Navigation, and Control Conference and Exhibit, Keystone, CO. 2006,.

[9] Atkin, J.A.D., Burke, E.K., Greenwood, J.S., Reeson, D.. Hybrid Metaheuristics to Aid Runway Scheduling at London Heathrow Airport. Transportation Science 2007;41(1):90-106. doi:10.1287/trsc.1060.0163.

[10] Atkin, J., Burke, E., Greenwood, J., Reeson, D.. On-line decision support for take-off runway scheduling with uncertain taxi times at London Heathrow airport. Journal of Scheduling 2008;11(5):323-346. doi:10.1007/s10951-008-0065-9. URL http://dx.doi.org/10.1007/s10951-008-0065-9.

[11] Hu, X.B., Di Paolo, E.. Binary-Representation-Based Genetic Algorithm for Aircraft Arrival Sequencing and Scheduling. Intelligent Transportation Systems, IEEE Transactions on 2008;9(2):301-310. doi:10.1109/TITS.2008.922884.

[12] Anagnostakis, I., Clarke, J.P.. Runway operations planning: a two-stage solution methodology. In: System Sciences, 2003. Proceedings of the 36th Annual Hawaii International Conference on. 2003, p. 12 pp.-. doi:10.1109/HICSS.2003.1174196.

[13] Bennell, J., Mesgarpour, M., Potts, C.. Airport runway scheduling. Annals of Operations Research 2013;204(1):249-270. doi:10.1007/s10479-012-1268-1. URL http://dx.doi.org/10.1007/s10479-012-1268-1.

[14] Atkin, J.A., Burke, E.K., Ravizza, S.. The airport ground movement problem: Past and current research and future directions. Proceedings of the 4th International Conference on Research in Air Transportation (ICRAT), Budapest, Hungary 2010;:131-138

[15] Pesic, B., Durand, N., Alliot, J.. Aircraft ground traffic optimisation using a genetic algorithm. In: Proceedings of the Genetic and Evolutionary Computation Conference (GECCO), San Francisco, California, USA. 2001, p. 1397-1404.

[16] Marín, A.. Airport management: taxi planning. Annals of Operations Research 2006;143(1):191-202. doi:10.1007/s10479006-7381-2.

[17] Roling, P.C., Visser, H.G.. Optimal Airport Surface Traffic Planning Using Mixed-Integer Linear Programming. International Journal of Aerospace Engineering 2008;vol. 2008:11.

[18] Ravizza, S., Atkin, J.A., Burke, E.K.. A more realistic approach for airport ground movement optimisation with stand holding. Journal of Scheduling 2013;:1-14doi:10.1007/s10951-013-0323-3.

[19] Lesire, C.. An Iterative A* Algorithm for Planning of Airport Ground Movements. ISBN 978-1-60750-605-8; ????,doi: 10.3233/978-1-60750-606-5-413. 19th European Conference on Artificial Intelligence (ECAI)/6th Conference on Prestigious Applications of Intelligent Systems (PAIS), Lisbon, Portugal, August 16-20, 2010.

[20] Balakrishnan, H., Jung, Y.. A framework for coordinated surface operations planning at Dallas-Fort Worth International Airport. In: Proceedings of the AIAA Guidance, Navigation, and Control Conference, Hilton Head, SC, USA. 2007,.

[21] Smeltink, J., Soomer, M., de Waal, P., van der Mei, R.. An optimisation model for airport taxi scheduling. In: Proceedings of the INFORMS Annual Meeting, Denver, Colorado, USA. 2004,.

[22] Marín, A., Codina, E.. Network design: taxi planning. Annals of Operations Research 2008;157(1):135-151. doi: $10.1007 / \mathrm{s} 10479-007-0194-0$

[23] Atkin, J.A., Burke, E.K., Greenwood, J.S.. TSAT allocation at London Heathrow: the relationship between slot compliance, throughput and equity. Public Transport 2010;2(3):173-198. doi:10.1007/s12469-010-0029-2.

[24] Atkin, J.A., Burke, E.K., Greenwood, J.S.. A comparison of two methods for reducing take-off delay at London Heathrow airport. Journal of Scheduling 2011;14(5):409-421. doi:10.1007/s10951-011-0228-y.

[25] Burgain, P., Feron, E., Clarke, J.. Collaborative virtual queue: Benefit analysis of a collaborative decision making concept applied to congested airport departure operations. Air Traffic Control Quarterly 2009;17(2):195-222.

[26] Ravizza, S., Chen, J., Atkin, J.A., Burke, E.K., Stewart, P.. The trade-off between taxi time and fuel consumption in airport ground movement. Public Transport 2013;5(1-2):25-40. doi:10.1007/s12469-013-0060-1.

[27] Chen, J., Stewart, P.. Planning aircraft taxiing trajectories via a multi-ojective immune optimisation. In: Natural Computation (ICNC), 2011 Seventh International Conference on; vol. 4. 2011, p. 2235-2240. doi:10.1109/ICNC.2011.6022587.

[28] Weiszer, M., Chen, J., Ravizza, S., Atkin, J., Stewart, P.. A heuristic approach to greener airport ground movement. 2014, p. 3280-3286. doi:10.1109/CEC.2014.6900372.

[29] Deau, R., Gotteland, J.B., Durand, N.. Runways sequences and ground traffic optimisation. In: ICRAT 2008, International Conference on Research in Air Transportation. Fairfax, USA; 2008, p. pp xxxx. URL http://hal-enac.archives-ouvertes.fr/hal-00940950.

[30] Deau, R., Gotteland, J., Durand, N.. Airport surface management and runways scheduling. In: Proceedings of the 8th USA/Europe Air Traffic Management Research and Development Seminar, Napa, CA, USA. 2009,. 
[31] Clare, G., Richards, A.. Optimization of Taxiway Routing and Runway Scheduling. Intelligent Transportation Systems, IEEE Transactions on 2011;12(4):1000-1013. doi:10.1109/TITS.2011.2131650.

[32] Frankovich, M., Bertsimas, D.. Air Traffic Flow Management at Airports: A Unified Optimization Approach. In: Tenth USA/EUROPE Air Traffic Management Research \& Development Seminar. 2013,

[33] Diepen, G., Pieters, B., van den Akker, J., Hoogeveen, J.. Robust planning of airport platform buses. Computers \& Operations Research 2013;40(3):747-757. doi:10.1016/j.cor.2011.08.002. Transport Scheduling; URL http://www.sciencedirect.com/science/article/pii/S0305054811002267.

[34] Ascó, A., Atkin, J., Burke, E.. An Evolutionary Algorithm for the Over-constrained Airport Baggage Sorting Station Assignment Problem. In: Bui, L., Ong, Y., Hoai, N., Ishibuchi, H., Suganthan, P., editors. Simulated Evolution and Learning; vol. 7673 of Lecture Notes in Computer Science. Springer Berlin Heidelberg. ISBN 978-3-642-34858-7; 2012, p. 32-41. doi:10.1007/978-3-642-34859-4_4. URL http://dx.doi.org/10.1007/978-3-642-34859-4_4.

[35] Ip, W., Wang, D., Cho, V.. Aircraft Ground Service Scheduling Problems and Their Genetic Algorithm With Hybrid Assignment and Sequence Encoding Scheme. Systems Journal, IEEE 2013;7(4):649-657. doi:10.1109/JSYST.2012.2196229.

[36] Du, Y., Zhang, Q., Chen, Q.. ACO-IH: An improved ant colony optimization algorithm for Airport Ground Service Scheduling. In: Industrial Technology, 2008. ICIT 2008. IEEE International Conference on. 2008, p. 1-6. doi: 10.1109/ICIT.2008.4608674.

[37] Ho, S.C., Leung, J.M.. Solving a manpower scheduling problem for airline catering using metaheuristics. European Journal of Operational Research 2010;202(3):903-921. doi:10.1016/j.ejor.2009.06.030. URL http://www.sciencedirect.com/science/article/pii/S0377221709004962.

[38] Kuhn, K., Loth, S.e.. Airport Service Vehicle Scheduling. In: Proceedings of the 8th USA/Europe Air Traffic Management Research and Development Seminar, Napa, CA, USA. 2009,.

[39] Neuman, U.M., Atkin, J.A.. Airport Gate Assignment Considering Ground Movement. In: Computational Logistics. Springer; 2013, p. 184-198.

[40] Bonyadi, M., Michalewicz, Z., Barone, L.. The travelling thief problem: The first step in the transition from theoretical problems to realistic problems. In: Evolutionary Computation (CEC), 2013 IEEE Congress on. 2013, p. 1037-1044. doi:10.1109/CEC.2013.6557681.

[41] Stolk, J., Mann, I., Mohais, A., Michalewicz, Z.. Combining vehicle routing and packing for optimal delivery schedules. OR Insight 2013;26(3):167-190. URL http://dx.doi.org/10.1057/ori.2013.1.

[42] Ibrahimov, M., Mohais, A., Schellenberg, S., Michalewicz, Z.. Evolutionary approaches for supply chain optimisation. Part II: multi-silo supply chains. International Journal of Intelligent Computing and Cybernetics 2012;5(4):473-499.

[43] Mei, Y., Li, X., Yao, X.. On investigation of interdependence between sub-problems of the Travelling Thief Problem. Soft Computing 2014;:1-16.

[44] Deb, K., Pratap, A., Agarwal, S., Meyarivan, T.. A fast and elitist multiobjective genetic algorithm: NSGA-II. Evolutionary Computation, IEEE Transactions on 2002;6(2):182-197. doi:10.1109/4235.996017.

[45] Frankovich, M.J.. Air traffic flow management at airports: a unified optimization approach. Ph.D. thesis; Massachusetts Institute of Technology; 2012.

[46] Chen, J., Weiszer, M.. Towards A More Cost Effective and Environmental Friendly Airport Surface Movement through Active Routing Part 1: Optimal Trajectory Generation; 2014.

[47] Nikoleris, T., Gupta, G., Kistler, M.. Detailed estimation of fuel consumption and emissions during aircraft taxi operations at Dallas/Fort Worth International Airport. Transportation Research Part D: Transport and Environment 2011;16(4):302-308. doi:10.1016/j.trd.2011.01.007.

[48] Freling, R., Wagelmans, A., Paixão, J.P.. Models and Algorithms for Single-Depot Vehicle Scheduling 2001;35:165-180.

[49] Jonker, R., Volgenant, A.. A Shortest Augmenting Path Algorithm for Dense and Sparse Linear Assignment Problems $1987 ; 38: 325-340$.

[50] Assaf, A.. Accounting for size in efficiency comparisons of airports. Journal of Air Transport Management 2009;15(5):256258.

[51] Eurocontrol, . European airline delay cost reference values.

2011.

URL https://www.eurocontrol.int/sites/default/files/publication/files/european-airline-delay-cost-reference-values-final-repo

[52] IATA, . Fuel Price Analysis. 2014. URL http://www .iata.org/publications/economics/fuel-monitor/Pages/price-analysis.aspx.

[53] Garrett, A.. Inspyred. 2012. (Version 1.0) [software]. Inspired Intelligence Initiative. Retrieved from http://inspyred.github.com. 